HEPHY-PUB 669/97

UWThPh-1997-13

hep-ph / 9705245

\title{
Electroweak dipole moment form factors of the top quark in supersymmetry
}

\author{
A. Bartl \\ Institut für Theoretische Physik, Universität Wien, \\ A-1090 Vienna, Austria \\ E. Christova \\ Institute of Nuclear Research and Nuclear Energy, \\ Boul. Tzarigradsko Chaussee 72, Sofia 1784, Bulgaria \\ T. Gajdosik, W. Majerotto \\ Institut für Hochenergiephysik der Österreichischen Akademie der Wissenschaften, \\ A-1050 Vienna, Austria
}

\begin{abstract}
The CP violating electric and weak dipole moment form factors of the top quark, $d^{\gamma}(s)$ and $d^{Z}(s)$, appear in the process $e^{+} e^{-} \rightarrow t \bar{t}$. We present a complete analysis of these dipole moment form factors within the Minimal Supersymmetric Standard Model with complex parameters. We include gluino, chargino, and neutralino exchange in the loop of the $\gamma t \bar{t}$ and $Z t \bar{t}$ vertex. We give the analytic expressions and present numerical results.
\end{abstract}




\section{Introduction}

Owing to its large mass [目] the top quark decays before its polarization is diluted by hadronization effects. This implies that one can keep track of its polarization by the distribution of its decay products. This property of the top quark offers new possibilities of testing existing models. New measurable quantities are provided by the polarization and the corresponding calculations can be performed perturbatively. The large mass of the top quark also allows one to probe physics at a high energy scale, where new physics might show up.

In the last years a number of papers [2, 3, 4] considered $\mathrm{CP}$ violating observables in top quark production as tests for new physics. In $e^{+} e^{-}$annihilation these effects are due to the weak $d^{Z}(s)$ and electric $d^{\gamma}(s)$ dipole moment form factors of the top. In general, the vertices including the $\mathrm{CP}$ violating form factors are

$$
\begin{aligned}
e\left(\mathcal{V}_{\gamma}^{t, \bar{t}}\right)_{\mu} & =e\left(\frac{2}{3} \gamma_{\mu} \mp i \frac{d^{\gamma}(s)}{m_{t}} \mathcal{P}_{\mu} \gamma_{5}\right), \\
\frac{g}{2 \cos \Theta_{W}}\left(\mathcal{V}_{Z}^{t, \bar{t}}\right)_{\mu} & =\frac{g}{2 \cos \Theta_{W}}\left(\gamma_{\mu}\left(g_{V} \pm g_{A} \gamma_{5}\right) \mp i \frac{d^{Z}(s)}{m_{t}} \mathcal{P}_{\mu} \gamma_{5}\right),
\end{aligned}
$$

where $\mathcal{P}_{\mu}=p_{t \mu}-p_{\bar{t} \mu}, g_{V}=(1 / 2)-(4 / 3) \sin ^{2} \Theta_{W}, g_{A}=-(1 / 2)$, and $g=e / \sin \Theta_{W}$ with $e$ the electro-magnetic coupling constant and $\Theta_{W}$ the Weinberg angle. The possibilities of the present experimental facility to measure different $\mathrm{CP}$ violating observables in processes with top quarks have been studied in [5]. As all CP violating observables that can be measured depend on these dipole moment form factors, precise predictions are necessary.

In the Standard Model (SM) CP violation can appear only through the phase in the CKM-matrix. The dipole moment form factors $d^{\gamma}(s)$ and $d^{Z}(s)$ for the quarks are at least two-loop order effects and hence very small. CP violating effects at one-loop level can arise from new interactions, and therefore may be larger. The possibility of observing CP violating effects in top physics is associated with new physics.

In this paper we analyse the supersymmetric (SUSY) contributions to $d^{\gamma}(s)$ and $d^{Z}(s)$ of the top quark. In the Minimal Supersymmetric Standard Model (MSSM) [6] additional complex couplings can be introduced that lead to $\mathrm{CP}$ violation within one generation only [7]. If the masses of the SUSY particles are not very much higher than the mass of the top, one expects SUSY radiative corrections to induce larger values for $d^{\gamma}(s)$ and $d^{Z}(s)$.

There is an essential difference between the dipole moments of the electron $d_{e}$ (or neutron $\left.d_{n}\right)$ and the dipole moment form factors $d^{\gamma}(s), d^{Z}(s)$ of the top: $d_{e}\left(d_{n}\right)$ measures the interaction of the stable electron (neutron) with almost free photons; $d^{\gamma}(s)$ and $d^{Z}(s)$ are form factors that measure the interaction of the short-lived top quark with an off-shell $\gamma$ or $Z$. The off-shellness of $\gamma$ and $Z$ leads to strong enhancements in $d^{\gamma}(s), d^{Z}(s)$ if the 
particles in the loop that couple to $\gamma, Z$ are near the threshold $\sqrt{s}=m_{j}+m_{k}$, with $m_{j}$, $m_{k}$ being the masses of these particles. According to the particle content in the loop we distinguish the following three contributions:

1. gluino contribution $d_{\tilde{g}}^{\gamma, Z}$ with $\left(\tilde{t} \tilde{t}^{*} \tilde{g}\right)$ in the loop,

2. chargino contribution $d_{\tilde{\chi}^{+}}^{\gamma, Z}$ with $\left(\tilde{\chi}^{+} \tilde{\chi}^{-} \tilde{b}\right)$ and $\left(\tilde{b} \tilde{b}^{*} \tilde{\chi}^{+}\right)$in the loop,

3. neutralino contribution $d_{\tilde{\chi}^{0}}^{\gamma, Z}$ with $\left(\tilde{\chi}^{0} \tilde{\chi}^{0} \tilde{t}\right)$ and $\left(\tilde{t} \tilde{t}^{*} \tilde{\chi}^{0}\right)$ in the loop.

The gluino contribution $d_{\tilde{g}}^{\gamma, Z}$ was considered in 3 , 国. In this article we consider the chargino and neutralino contributions. Although the gluino contribution $d_{\tilde{g}}^{\gamma, Z}$ is proportional to $\alpha_{s}$ we show that the chargino contribution $d_{\tilde{\chi}^{+}}^{\gamma, Z}$, which is proportional to $\alpha_{w}$, $\left(\alpha_{w}=g^{2} /(4 \pi)\right)$ can be equally important. This is due to threshold enhancements and the large Yukawa couplings: $Y_{t}=m_{t} /\left(\sqrt{2} m_{W} \sin \beta\right)$ and $Y_{b}=m_{b} /\left(\sqrt{2} m_{W} \cos \beta\right)$. In general the neutralino contribution turns out to be smaller. However, there are cases where it is important. The mass spectra of the SUSY particles are crucial for the understanding of the electroweak dipole moment form factors.

In order to obtain a non-zero dipole moment one needs both an operator changing the helicity of the top and a mixture of the interaction eigenstates of the squarks, charginos, and neutralinos, which provide a CP violating phase. In the MSSM the CP violating phases for the chargino and neutralino contribution are provided by the chargino and neutralino mass matrices and the scalar quark mass mixing matrices. The calculation requires the diagonalization of all these matrices. The chargino mass matrix with complex phases has been diagonalized analytically in [8]. In the present paper we use the singular value decomposition [9] to diagonalize the complex neutralino and chargino mass matrices.

The size of the dipole moment form factors $d^{\gamma}(s), d^{Z}(s)$ depends strongly on the phases of the SUSY parameters. There are constraints [8, 10, 11] on some phases from the measurement of the EDM of the neutron. Usually, one concludes [8, 12] that either the phases involved in the EDM of the neutron are very small or the masses of the first generation of squarks are in the $\mathrm{TeV}$ range. By using supergravity (SUGRA) with grand unification (GUT) there are attempts to constrain also the phases entering the dipole moments of the top [10, 11]. In our analysis we want to be more general and we do not make any additional assumptions about GUT except for the unification of the gauge couplings. In particular, we do not assume unification of the scalar mass parameters and the trilinear scalar coupling parameters $A_{q}$ of the different generations. As the breaking mechanism of SUSY is not known, a definite theoretical ansatz about the size of the complex phases involved in the weak and electric dipole moment form factors of the top quark cannot be given. An unambigous decision will be provided by experiment. Therefore, measuring the weak and electric dipole moment form factors of the top quark might be also a test 
of the unification assumption involved and could give insight into the SUSY breaking mechanism.

In this paper we present a complete analysis of the weak and electric dipole moment form factors of the top quark within the MSSM with complex parameters. We give the analytic expressions and present numerical results.

The paper is organized as follows. In section 2 we summarize the parts of the Lagrangian that are relevant, and fix the notation. Generic formulae for contributions to the dipole moment form factors are given in section 3. In section 4 we present the chargino contribution, in section 5 follows the neutralino contribution. The numerical analysis is performed in section 6 . The conclusions are given in section 7 .

\section{The SUSY Lagrangian with complex couplings}

In the MSSM with complex phases, $d^{\gamma}(s)$ and $d^{Z}(s)$ are generated in one-loop order, irrespectively of generation mixing. The gluino contribution to the electroweak dipole moment form factors was previously studied in [4]. Here we discuss the chargino and neutralino contributions. These contributions have to be treated separately from the gluino contribution not only because different couplings are involved (electroweak and strong), but also because they are sensitive to different SUSY parameters. The contributions from the different Feynman diagrams (Fig. 1a,b) depend in a distinctive way on the SUSY parameters. For the sake of clarity we discuss the chargino and neutralino contributions separately.

The parts of the Lagragian that contribute to the electric and weak dipole moment form factors of the top are:

$$
\begin{aligned}
\mathcal{L}_{\gamma \tilde{\chi}_{j}^{+} \tilde{\chi}_{k}^{-}} & =-e A_{\mu} \delta_{j k} \overline{\tilde{\chi}}_{j}^{+} \gamma^{\mu} \tilde{\chi}_{k}^{+} \\
\mathcal{L}_{\gamma \tilde{q}_{m} \tilde{q}_{n}} & =-i e Q_{\tilde{q}} A_{\mu} \delta_{m n}\left(\tilde{q}_{m}^{*} \overleftrightarrow{\partial}^{\mu} \tilde{q}_{n}\right) \\
\mathcal{L}_{Z \tilde{\chi}_{j}^{+} \tilde{\chi}_{k}^{-}} & =g /\left(2 \cos \Theta_{W}\right) Z_{\mu} \tilde{\bar{\chi}}_{j}^{+} \gamma^{\mu}\left(O_{j k}^{L} P_{L}+O_{j k}^{R} P_{R}\right) \tilde{\chi}_{k}^{+} \\
\mathcal{L}_{Z \tilde{\chi}_{j}^{0} \tilde{\chi}_{k}^{0}} & =g /\left(2 \cos \Theta_{W}\right) Z_{\mu} \overline{\tilde{\chi}}_{j}^{0} \gamma^{\mu}\left(O_{j k}^{\prime \prime} P_{L}-O_{j k}^{\prime \prime *} P_{R}\right) \tilde{\chi}_{k}^{0} \\
\mathcal{L}_{Z \tilde{q}_{m} \tilde{q}_{n}} & =i g /\left(2 \cos \Theta_{W}\right) Z_{\mu} c_{m n}^{\tilde{q}}\left(\tilde{q}_{m}^{*} \overleftrightarrow{\partial}^{\mu} \tilde{q}_{n}\right) \\
\mathcal{L}_{\bar{q} \tilde{\chi}_{k}^{0} \tilde{q}_{m}} & =g \bar{q}\left(a_{m k}^{\tilde{q}} P_{R}+b_{m k}^{\tilde{q}} P_{L}\right) \tilde{\chi}_{k}^{0} \tilde{q}_{m} \\
\mathcal{L}_{q^{\prime} \tilde{\chi}_{k}^{+} \tilde{q}_{m}} & =g \bar{q}^{\prime}\left(l_{m k}^{\tilde{q}} P_{R}+k_{m k}^{\tilde{q}} P_{L}\right) \tilde{\chi}_{k}^{+} \tilde{q}_{m} \\
\mathcal{L}_{\tilde{q} \tilde{q} \tilde{q}_{m}} & =-g_{s}\left(\lambda_{u v}^{b} / \sqrt{2}\right) \bar{q}_{u}\left(e^{\frac{i}{2} \varphi_{\tilde{q}}}\left(\mathcal{R}^{\tilde{q}}\right)_{m L}^{*} P_{R}-e^{-\frac{i}{2} \varphi_{\tilde{q}}}\left(\mathcal{R}^{\tilde{q}}\right)_{m R}^{*} P_{L}\right) \tilde{g}^{b} \tilde{q}_{m v}
\end{aligned}
$$

In eq. (10) $\lambda^{b}$ are the Gell-Mann matricies, $u, v$ are the color indicies, and $\varphi_{\tilde{g}}$ is the phase of the gluino mass term. The couplings are defined as in [13]:

$$
O_{j k}^{R}=-\left(\cos 2 \Theta_{W} \delta_{j k}+U_{j 1}^{*} U_{k 1}\right) \quad O_{j k}^{L}=-\left(\cos 2 \Theta_{W} \delta_{j k}+V_{j 1} V_{k 1}^{*}\right)
$$




$$
\begin{aligned}
& O_{j k}^{\prime \prime}=-\frac{1}{2}\left[\cos 2 \beta\left(N_{3 j}^{*} N_{3 k}-N_{4 j}^{*} N_{4 k}\right)+\sin 2 \beta\left(N_{3 j}^{*} N_{4 k}+N_{4 j}^{*} N_{3 k}\right)\right]=O_{k j}^{\prime \prime *} \\
& c_{m n}^{\tilde{q}}=2 Q_{\tilde{q}} \sin ^{2} \Theta_{W} \delta_{m n}-2 \mathrm{I}_{3}^{\tilde{q}}\left(\mathcal{R}^{\tilde{q}}\right)_{m L}\left(\mathcal{R}^{\tilde{q}}\right)_{n L}^{*} \\
& a_{m j}^{\tilde{t}}=\mathcal{R}_{m L}^{\tilde{t *}} f_{L j}+\mathcal{R}_{m R}^{\tilde{t} *} h_{R j} \quad b_{m j}^{\tilde{t}}=\mathcal{R}_{m L}^{\tilde{t} *} h_{L j}+\mathcal{R}_{m R}^{\tilde{t} *} f_{R j} \\
& h_{L j}=Y_{t}\left(\sin \beta N_{3 j}-\cos \beta N_{4 j}\right) \quad h_{R j}=Y_{t}\left(\sin \beta N_{3 j}^{*}-\cos \beta N_{4 j}^{*}\right)=h_{L j}^{*} \\
& f_{L j}=-\left[\frac{2}{3} \sin 2 \Theta_{W} N_{1 j}^{*}+\left(g_{V}-g_{A}\right) N_{2 j}^{*}\right] /\left(\sqrt{2} \cos \Theta_{W}\right) \\
& f_{R j}=\left[\frac{2}{3} \sin 2 \Theta_{W} N_{1 j}+\left(g_{V}+g_{A}\right) N_{2 j}\right] /\left(\sqrt{2} \cos \Theta_{W}\right) \\
& l_{m j}^{\tilde{b}}=-\mathcal{R}_{m L}^{\tilde{b} *} U_{j 1}+\mathcal{R}_{m R}^{\tilde{b} *} Y_{b} U_{j 2} \quad k_{m j}^{\tilde{b}}=\mathcal{R}_{m L}^{\tilde{b} *} Y_{t} V_{j 2}^{*} \\
& \mathcal{R}^{\tilde{q}}=\left(\begin{array}{ll}
\mathcal{R}_{1 L}^{\tilde{q}} & \mathcal{R}_{1 R}^{\tilde{q}} \\
\mathcal{R}_{2 L}^{\tilde{q}} & \mathcal{R}_{2 R}^{\tilde{q}}
\end{array}\right)=\left(\begin{array}{cc}
e^{\frac{i}{2} \varphi_{\tilde{q}}} \cos \theta_{\tilde{q}} & e^{-\frac{i}{2} \varphi_{\tilde{q}}} \sin \theta_{\tilde{q}} \\
-e^{\frac{i}{2} \varphi_{\tilde{q}}} \sin \theta_{\tilde{q}} & e^{-\frac{i}{2} \varphi_{\tilde{q}}} \cos \theta_{\tilde{q}}
\end{array}\right),
\end{aligned}
$$

so that $\left(\begin{array}{c}\tilde{q}_{1} \\ \tilde{q}_{2}\end{array}\right)=\mathcal{R}^{\tilde{q}}\left(\begin{array}{l}\tilde{q}_{L} \\ \tilde{q}_{R}\end{array}\right) \cdot Q_{\tilde{q}}$ is the charge of $\tilde{q}$ and $\tan \beta=\frac{v_{2}}{v_{1}}$, where $v_{1}$ and $v_{2}$ are the vacuum expectations values of the Higgs fields, $P_{L, R}$ are the chirality projection operators.

The unitary matrices $U$ and $V$ diagonalize the chargino mass matrix:

$$
M_{\alpha \beta}^{\tilde{\chi}^{+}}=\left(\begin{array}{cc}
M & m_{W} \sqrt{2} \sin \beta \\
m_{W} \sqrt{2} \cos \beta & \mu
\end{array}\right), \quad U_{j \alpha}^{*} M_{\alpha \beta}^{\tilde{\chi}^{+}} V_{k \beta}^{*}=m_{\tilde{\chi}_{j}^{+}} \delta_{j k} .
$$

For the diagonalization of the chargino mass matrix one has to use the singular value decomposition [9]. The explicit procedure to obtain $U$ and $V$ is given in Appendix A.

$N_{\alpha k}$ is the unitary matrix which makes the complex symmetric neutralino mass matrix diagonal with positive diagonal elements [14]:

$$
N_{\alpha j} M_{\alpha \beta}^{\tilde{\chi}^{0}} N_{\beta k}=m_{\tilde{\chi}_{j}^{0}} \delta_{j k} .
$$

In the basis

$$
\psi_{\alpha}=\left\{-i \tilde{\gamma},-i \tilde{Z}, \tilde{H}_{1}, \tilde{H}_{2}\right\} \quad \text { with }\left\{\begin{array}{c}
\tilde{H}_{1}=\tilde{H}_{1}^{0} \cos \beta-\tilde{H}_{2}^{0} \sin \beta \\
\tilde{H}_{2}=\tilde{H}_{1}^{0} \sin \beta+\tilde{H}_{2}^{0} \cos \beta
\end{array}\right.
$$

used in [6], the complex symmetric neutralino mass matrix has the form

$$
M_{\alpha \beta}^{\tilde{\chi}^{0}}=\left(\begin{array}{cccc}
m_{\tilde{\gamma}} & m_{a z} & 0 & 0 \\
m_{a z} & m_{\tilde{z}} & m_{Z} & 0 \\
0 & m_{Z} & \mu \sin 2 \beta & -\mu \cos 2 \beta \\
0 & 0 & -\mu \cos 2 \beta & -\mu \sin 2 \beta
\end{array}\right)
$$


where

$$
\begin{aligned}
m_{\tilde{\gamma}} & =M \sin ^{2} \Theta_{W}+M^{\prime} \cos ^{2} \Theta_{W} \\
m_{\tilde{z}} & =M \cos ^{2} \Theta_{W}+M^{\prime} \sin ^{2} \Theta_{W} \\
m_{a z} & =\sin \Theta_{W} \cos \Theta_{W}\left(M-M^{\prime}\right)
\end{aligned}
$$

Here $M$ and $M^{\prime}$ are the $\mathrm{SU}(2)$ and $\mathrm{U}(1)$ gaugino masses, $\mu=|\mu| e^{i \varphi_{\mu}}$ is the mass parameter in front of the Higgs superfields in the Lagrangian. The procedure for obtaining $N_{\alpha k}$ is described in Appendix B. Again the singular value decomposition has to be used.

\section{General formula}

Technically there are two types of one-loop SUSY diagrams that contribute to the electric and weak dipole moments of the top quark, one with two scalars and one fermion in the loop as shown in Fig. 1a, and the other with two fermions and one scalar in the loop, Fig. 1b. Here we give generic formulae for the dipole moment form factors of the top induced by these graphs. The different combinations of Passarino-Veltman functions give different types of threshold enhancements.

We use a generic Lagrangian of the form

$$
\begin{aligned}
\mathcal{L} & =g_{1} V_{\mu} \bar{f}_{j} \gamma^{\mu}\left(O_{j k}^{L} P_{L}+O_{j k}^{R} P_{R}\right) f_{k}+i g_{1} V_{\mu} \Gamma_{m n}\left(s_{m}^{*} \stackrel{\leftrightarrow}{\partial}^{\mu} s_{n}\right) \\
& +g \bar{f}\left(a_{m k} P_{R}+b_{m k} P_{L}\right) f_{k} s_{m}+h . c .
\end{aligned}
$$

where $f_{i}$ stands for a fermion field, $s_{m}$ for a scalar field, and $V_{\mu}$ for a neutral vector boson field. The couplings $\Gamma_{m n}$ and $O_{j k}^{L, R}$ are hermitian. The couplings $a_{m k}$ and $b_{m k}$ are complex numbers. Fig. 1a then gives the following contribution:

$$
\begin{aligned}
d_{f f s}= & \alpha_{w} /(8 \pi) \times\left(f_{1}+f_{2}\right) \\
f_{1}= & -2 \sum_{m, j, k} m_{j} C_{1}^{m, j k} \Im \mathrm{m}\left[a_{m j} O_{j k}^{L} b_{m k}^{*}-b_{m j} O_{j k}^{R} a_{m k}^{*}\right] \\
f_{2}= & m_{t} \sum_{m, j, k}\left(C_{1}^{m, j k}+C_{11}^{m, j k}-C_{2}^{m, j k}-C_{22}^{m, j k}\right) \\
& \quad \times \Im \mathrm{m}\left[a_{m j} O_{j k}^{R} a_{m k}^{*}-b_{m j} O_{j k}^{L} b_{m k}^{*}\right]
\end{aligned}
$$

with $g^{2}=4 \pi \alpha_{w}$. Fig 1 b leads to

$$
\begin{aligned}
d_{s s f}= & \alpha_{w} /(8 \pi) \times\left(s_{1}+s_{2}\right) \\
s_{1}= & -2 \sum_{k, m, n} m_{k}\left(C_{0}^{k, m n}+C_{1}^{k, m n}+C_{2}^{k, m n}\right) \Im m\left[a_{m k} \Gamma_{m n} b_{n k}^{*}\right] \\
s_{2}= & -m_{t} \sum_{k, m, n}\left(C_{1}^{k, m n}+C_{11}^{k, m n}-C_{2}^{k, m n}-C_{22}^{k, m n}\right) \\
& \times \Im \mathrm{m}\left[a_{m k} \Gamma_{m n} a_{n k}^{*}-b_{m k} \Gamma_{m n} b_{n k}^{*}\right]
\end{aligned}
$$


For the Passarino-Veltman three point functions [15] $C_{0}, C_{i}$, and $C_{i i}(i=1,2)$ we follow the convention of [16]. They are defined in Appendix C. Note that there are only three types of contributions: $f_{2}$ and $s_{2}$ have the same functional form.

\section{Chargino contribution to $d^{\gamma}(s)$ and $d^{Z}(s)$}

Here we show the explicit dependence of the chargino contribution on the gaugino and higgsino couplings, as well as the dependence on the squark mixing angle and phase. For the electric dipole moment form factor we have:

$$
\begin{aligned}
\frac{d_{\tilde{\chi}^{+}}^{\gamma}}{m_{t}}=-\frac{\alpha_{e m} Y_{t}}{4 \pi \sin ^{2} \Theta_{W}} \sum_{m, i=1}^{2} m_{\tilde{\chi}_{i}^{+}}\left(C_{1}^{m, i i}-\frac{1}{6}\left(C_{0}^{i, m m}+C_{1}^{i, m m}+C_{2}^{i, m m}\right)\right) \times \\
\quad\left[\left(1-(-)^{m} \cos 2 \theta_{\tilde{b}}\right) \Im m\left[U_{i 1} V_{i 2}\right]+Y_{b}(-)^{m} \sin 2 \theta_{\tilde{b}} \Im \mathrm{m}\left[U_{i 2} V_{i 2} e^{i \varphi_{\tilde{b}}}\right]\right]
\end{aligned}
$$

The chargino contribution $d_{\tilde{\chi}^{+}}^{Z}$ to the weak dipole moment form factor of the top quark is:

$$
\begin{aligned}
\frac{d_{\tilde{\chi}^{+}}^{Z}}{m_{t}}= & \frac{\alpha_{e m}}{8 \pi \sin ^{2} \Theta_{W}}\left(f_{1}+f_{2}+s_{1}+s_{2}\right) \\
f_{1}= & -Y_{t} \sum_{m, j, k=1}^{2} m_{\tilde{\chi}_{j}^{+}} C_{1}^{m, j k} \times\left[\left(\delta_{j k}\left(1+2 \cos 2 \Theta_{W}\right)+\left|U_{k 1}\right|^{2}-\left|V_{k 2}\right|^{2}\right) \cdot\right. \\
& \left(\left(1-(-)^{m} \cos 2 \theta_{\tilde{b}}\right) \Im \mathrm{m}\left[U_{j 1} V_{j 2}\right]+Y_{b}(-)^{m} \sin 2 \theta_{\tilde{b}} \Im \mathrm{m}\left[U_{j 2} V_{j 2} e^{i \varphi_{\tilde{b}}}\right]\right) \\
& \left.-Y_{b}\left(1-\delta_{j k}\right)(-)^{m} \sin 2 \theta_{\tilde{b}} \Im \mathrm{m}\left[U_{j 2} V_{j 2} e^{i \varphi_{\tilde{b}}}\right]\right] \\
f_{2}= & -m_{t} Y_{b} \sin 2 \theta_{\tilde{b}} \Im \mathrm{m}\left[U_{11}^{*} U_{12} e^{i \varphi_{\tilde{b}}}\right] \sum_{m=1}^{2}(-)^{m}\left(C_{1}^{m, 12}+C_{11}^{m, 12}-C_{2}^{m, 12}-C_{22}^{m, 12}\right) \\
s_{1}= & -Y_{t} \sum_{k, m, n=1}^{2} m_{\tilde{\chi}}^{+}\left(C_{0}^{k, m n}+C_{1}^{k, m n}+C_{2}^{k, m n}\right) \\
& \quad \times\left(\frac{2}{3} \sin ^{2} \Theta_{W} \delta_{m n}+\frac{1}{2}\left(1-(-)^{n} \cos 2 \theta_{\tilde{b}}\right)\right) \\
& \quad\left[\left(\left(1-(-)^{m} \cos 2 \theta_{\tilde{b}}\right) \Im \mathrm{m}\left[U_{k 1} V_{k 2}\right]+Y_{b}(-)^{m} \sin 2 \theta_{\tilde{b}} \Im \mathrm{m}\left[U_{k 2} V_{k 2} e^{\left.i \varphi_{\tilde{b}}\right]}\right]\right]\right. \\
s_{2}= & -m_{t} Y_{b} \sin 2 \theta_{\tilde{b}} \sum_{k=1}^{2}\left(C_{1}^{k, 12}+C_{11}^{k, 12}-C_{2}^{k, 12}-C_{22}^{k, 12}\right) \times \Im \mathrm{m}\left[U_{k 1}^{*} U_{k 2} e^{i \varphi_{\tilde{b}}}\right]
\end{aligned}
$$

We have included the terms proportional to the bottom Yukawa coupling $Y_{b}$ which are important for large $\tan \beta$. For small values of $\tan \beta$ the terms proportional to $Y_{t}$ dominate. The exchange of the lighter scalar bottom (see Fig. 1a) gives the leading contribution $\left(\cos 2 \theta_{\tilde{b}} \approx 1\right)$. Note that there are no terms proportional to $Y_{t}^{2}$ or $Y_{b}^{2}$. 


\section{Neutralino contribution to $d^{\gamma}(s)$ and $d^{Z}(s)$}

As neutralinos do not couple to the photon, $d_{\tilde{\chi}^{0}}^{\gamma}$ receives a non-zero contribution only from the diagram with $\tilde{t} \tilde{t}^{*} \tilde{\chi}^{0}$ exchanged in the loop. We have:

$$
\begin{aligned}
& \frac{d_{\tilde{\chi}^{0}}^{\gamma}}{m_{t}}=\frac{\alpha_{e m}}{12 \pi \sin ^{2} \Theta_{W}} \sum_{k=1}^{4} \sum_{m=1}^{2} m_{\tilde{\chi}_{k}^{+}}\left(C_{0}^{k, m m}+C_{1}^{k, m m}+C_{2}^{k, m m}\right) \\
& \times\left[(-)^{m} \sin 2 \theta_{\tilde{t}} \Im \mathrm{m}\left[\left(h_{L k}^{2}-f_{L k} f_{R k}^{*}\right) e^{-i \varphi_{\tilde{t}}}\right]\right. \\
& \left.-\left(1-(-)^{m} \cos 2 \theta_{\tilde{t}}\right) \Im \mathrm{m}\left[h_{L k} f_{L k}^{*}\right]-\left(1+(-)^{m} \cos 2 \theta_{\tilde{t}}\right) \Im \mathrm{m}\left[h_{L k} f_{R k}\right]\right]
\end{aligned}
$$

The neutralino contribution to $d^{Z}(s)$ is

$$
\begin{aligned}
& \frac{d_{\tilde{\chi}^{0}}^{Z}}{m_{t}}=\frac{\alpha_{e m}}{8 \pi \sin ^{2} \Theta_{W}}\left(2 f_{1}+2 f_{2}+s_{1}+s_{2}\right) \\
& f_{1}=\frac{1}{2} \sum_{j, k=1}^{4} \sum_{m=1}^{2} m_{\tilde{\chi}_{j}^{0}} C_{1}^{m, j k} \times\left[(-)^{m} \sin 2 \theta_{\tilde{t}} \Im \mathrm{m}\left[O_{j k}^{\prime \prime}\left(f_{L j} f_{R k}^{*}-f_{L k} f_{R j}^{*}\right) e^{-i \varphi_{\tilde{t}}}\right]\right. \\
& -\left(1+(-)^{m} \cos 2 \theta_{\tilde{t}}\right) \Im \mathrm{m}\left[O_{j k}^{\prime \prime}\left(h_{L j}^{*} f_{R k}^{*}-h_{L k}^{*} f_{R j}^{*}\right)\right] \\
& \left.-\left(1-(-)^{m} \cos 2 \theta_{\tilde{t}}\right) \Im \mathrm{m}\left[O_{j k}^{\prime \prime}\left(h_{L j} f_{L k}^{*}-h_{L k} f_{L j}^{*}\right)\right]\right] \\
& f_{2}=\frac{1}{2} m_{t} \sum_{j<k}^{4} \sum_{m=1}^{2}\left(C_{1}^{m, j k}+C_{11}^{m, j k}-C_{2}^{m, j k}-C_{22}^{m, j k}\right) \\
& \times\left[2(-)^{m} \cos 2 \theta_{\tilde{t}} \Im \mathrm{m}\left[h_{L j} O_{j k}^{\prime \prime} h_{L k}^{*}\right]\right. \\
& -2(-)^{m} \sin 2 \theta_{\tilde{t}} \Im \mathrm{m}\left[\left(f_{L j}^{*}-f_{R j}\right) O_{j k}^{\prime \prime} h_{L k}^{*} e^{i \varphi_{\tilde{t}}}\right] \\
& +\left(1-(-)^{m} \cos 2 \theta_{\tilde{t}}\right) \Im^{m}\left[f_{L j}^{*} O_{j k}^{\prime \prime} f_{L k}\right] \\
& \left.+\left(1+(-)^{m} \cos 2 \theta_{\tilde{t}}\right) \Im \mathrm{m}\left[f_{R j} O_{j k}^{\prime \prime} f_{R k}^{*}\right]\right] \\
& s_{1}=\frac{1}{2} \sum_{k=1}^{4} \sum_{m, n=1}^{2} m_{\tilde{\chi}_{k}^{+}}\left(C_{0}^{k, m n}+C_{1}^{k, m n}+C_{2}^{k, m n}\right) \\
& \times\left[(-)^{m}\left(\frac{8}{3} \sin ^{2} \Theta_{W} \delta_{m n}-\left(1-(-)^{n} \cos 2 \theta_{\tilde{t}}\right)\right) \Im m\left[\left(h_{L k}^{2}-f_{L k} f_{R k}^{*}\right) e^{-i \varphi_{\tilde{t}}}\right]\right. \\
& -\left(\frac{8}{3} \sin ^{2} \Theta_{W} \delta_{m n}\left(1+(-)^{m} \cos 2 \theta_{\tilde{t}}\right)-(-)^{m+n} \sin ^{2} 2 \theta_{\tilde{t}}\right) \Im \mathrm{m}\left[h_{L k} f_{R k}\right] \\
& \left.-\left(\frac{8}{3} \sin ^{2} \Theta_{W} \delta_{m n}-\left(1-(-)^{n} \cos 2 \theta_{\tilde{t}}\right)\right)\left(1-(-)^{m} \cos 2 \theta_{\tilde{t}}\right) \Im \mathrm{m}\left[h_{L k} f_{L k}^{*}\right]\right] \\
& s_{2}=m_{t} \sin 2 \theta_{\tilde{t}} \sum_{k=1}^{4}\left(C_{1}^{k, 12}+C_{11}^{k, 12}-C_{2}^{k, 12}-C_{22}^{k, 12}\right) \Im \mathrm{m}\left[h_{L k}^{*}\left(f_{L k}^{*}-f_{R k}\right) e^{i \varphi_{\tilde{t}}}\right]
\end{aligned}
$$

$f_{L j}, f_{R k}$ are gaugino couplings and $h_{L j}$ are higgsino couplings that contain the large Yukawa coupling $Y_{t}$. Notice that the factor of 2 in front of $f_{1}$ and $f_{2}$ in eq.(33) is due to the Majorana nature of the neutralinos. 


\section{$6 \quad$ Numerical results}

In this section we give numerical results for the real and imaginary parts of $d^{\gamma}(s)$ and $d^{Z}(s)$. Quite generally they depend on the parameters $M^{\prime}, M,|\mu|, \tan \beta, m_{\tilde{t}_{k}}, m_{\tilde{b}_{k}}, \cos \theta_{\tilde{t}}$, $\cos \theta_{\tilde{b}}$ and the phases $\varphi_{\mu}, \varphi_{\tilde{t}}, \varphi_{\tilde{b}}$, and $\varphi_{\tilde{g}}$. The GUT relations

$$
\begin{aligned}
m_{\tilde{g}} & =\left(\alpha_{s} / \alpha_{2}\right) M \approx 3 M \\
M^{\prime} & =\frac{5}{3} \tan ^{2} \Theta_{W} M
\end{aligned}
$$

imply that the gaugino mass parameters have the same phase. This phase can be removed by a $\mathrm{R}$-transformation [7].

We take $m_{W}=80 \mathrm{GeV}, m_{t}=175 \mathrm{GeV}, m_{b}=5 \mathrm{GeV}, \sqrt{s}=500 \mathrm{GeV}, \alpha_{s}=0.1$, and $\alpha_{e m}=\frac{1}{123}$. In order not to vary too many parameters we choose a reference set of parameter values given in the following table:

\begin{tabular}{rcc|rrrrr|}
$M$ & $=$ & $230,360 \mathrm{GeV}$ & $m_{\tilde{t}_{1}}$ & $=$ & $150 \mathrm{GeV}$ & $m_{\tilde{b}_{1}}$ & $=270 \mathrm{GeV}$ \\
$|\mu|$ & $=$ & $250 \mathrm{GeV}$ & $m_{\tilde{t}_{2}}$ & $=400 \mathrm{GeV}$ & $m_{\tilde{b}_{2}}$ & $=280 \mathrm{GeV}$ \\
$\tan \beta$ & $=$ & 2 & $\theta_{\tilde{t}}$ & $=$ & $\frac{\pi}{9}$ & $\theta_{\tilde{b}}$ & $=\frac{\pi}{36}$ \\
$\varphi_{\mu}$ & $=$ & $\frac{4 \pi}{3}$ & $\varphi_{\tilde{t}}$ & $=\frac{\pi}{6}$ & $\varphi_{\tilde{b}}$ & $=\frac{\pi}{3}$ \\
\hline
\end{tabular}

For $\tan \beta=2$ the terms proportional to $Y_{b}$ are strongly suppressed, and as one can easily verify from the explicit expressions (27)-(31) for $d_{\tilde{\chi}^{+}}^{\gamma, Z}$, the result will be independent on the phase $\varphi_{\tilde{b}}$. Thus $d^{\gamma}(s)$ and $d^{Z}(s)$ will depend on two phases: $\varphi_{\mu}$ and $\varphi_{\tilde{t}}$.

Notice that the dipole moment form factors $d^{\gamma}(s)$ and $d^{Z}(s)$ depend on $\varphi_{\mu}$ not only through the mixing matrices $U, V$, and $N$ in the couplings, but also through the chargino and neutralino mass spectra. In Fig. $2 \mathrm{a}$ and $\mathrm{b}$ we show the dependence of the chargino and neutralino masses on the phase $\varphi_{\mu}$ for $M=230 \mathrm{GeV}$ and the other parameters as in the reference set. As can be seen the values of the masses vary by about 40 percent in the whole $\cos \varphi_{\mu}$ region.

Next we study the dependence of $d^{\gamma}(s)$ and $d^{Z}(s)$ on the parameters $M$ and $|\mu|$ which control the chargino and neutralino masses and couplings. In Fig. 3a we show the $|\mu|$ dependence of the chargino contributions to $\Im \mathrm{m} d^{\gamma}(s)$ and $\Im \mathrm{m} d^{Z_{(}}(s)$ keeping the other parameters fixed at the reference values.

$\Im m d^{\gamma}(s)$ and $\Im \mathrm{m} d^{Z}(s)$ are determined by the absorptive parts of the amplitudes. Therefore they vanish when no real production of charginos is possible, i.e. $\sqrt{s} \leq 2 m_{\tilde{\chi}_{1}^{+}}$ as can be seen from the dotted curve of Fig. 3a. Local maxima occur near the thresholds of chargino pair production and they get bigger if the gaugino and higgsino component of the chargino are approximately equal. The $\tilde{\chi}_{1}^{+} \tilde{\chi}_{1}^{-}$-contribution has always the opposite sign of the $\tilde{\chi}_{2}^{+} \tilde{\chi}_{2}^{-}$-contribution because of the coupling $\Im m\left(U_{i 1} V_{i 2}\right)$ (see eq.(27)). 
In Fig. 4a we show the neutralino contribution to $\Im \mathrm{m} d^{\gamma}(s)$ and $\Im \mathrm{m} d^{Z}(s)$. $\Im \mathrm{m} d_{\tilde{\chi}^{0}}^{\gamma}$ is one order of magnitude smaller than the chargino contribution because the photon does not couple to the neutralinos and only Fig. 1 b contributes. The neutralino contribution $\Im m d_{\tilde{\chi}^{0}}^{Z}$ is smaller than the chargino contribution because the couplings are smaller. It shows the same qualitative behaviour as the chargino contributions, but it is more complicated because of the richer particle spectrum. Note that the two neutralinos in Fig. 1a have to be different.

In Fig. 3b we show the chargino contribution to $\Re$ e $d^{\gamma}(s)$ and $\Re$ e $d^{Z}(s)$ as a function of $|\mu|$ for $M=230,360 \mathrm{GeV}$ and the other parameters as given in the reference set. The behaviour of $\Re$ e $d_{\tilde{\chi}^{+}}^{\gamma}$ and $\Re$ e $d_{\tilde{\chi}^{+}}^{Z}$ can be understood by the dispersion relations by which $\Re$ e $d^{\gamma}(s)$ and $\Re$ e $d^{Z}(s)$ are related to the absorptive parts of $d^{\gamma}(s)$ and $d^{Z}(s)$. In Fig. 4b we show the neutralino contribution $\Re$ e $d_{\tilde{\chi}^{0}}^{Z}$. As one can see it has an analogous behaviour as the chargino contribution (Fig. 3b), but it is smaller.

In Fig. 5a we show the dependence of the chargino contribution $\Im m d_{\tilde{\chi}^{+}}^{\gamma}$ and $\Im m d_{\tilde{\chi}^{+}}^{Z}$ on the phase $\varphi_{\mu}$. Note that for $M=360 \mathrm{GeV} \Im m d_{\tilde{\chi}^{+}}^{\gamma}$ and $\Im m d_{\tilde{\chi}^{+}}^{Z}$ vanish in the intervall $0.82 \pi<\left|\varphi_{\mu}\right|<1.18 \pi$. This reflects the dependence of the chargino masses on the phase $\varphi_{\mu}$ (Fig. 2a): $m_{\tilde{\chi}_{1}^{+}}$decreases with $\cos \varphi_{\mu}$, and for $0.82 \pi<\left|\varphi_{\mu}\right|<1.18 \pi$ we are below the threshold: $\sqrt{s} \leq 2 m_{\tilde{\chi}_{1}^{+}}$. The maxima of $\Im m d^{\gamma}(s)$, $\varsigma m d^{Z}(s)$ for the parameters used appear at $\varphi_{\mu}=0.65 \pi$, and $\varphi_{\mu}=0.40 \pi$, and not at $\varphi_{\mu}=0.5 \pi$ as one would naively expect.

In Fig. 5c one can clearly see the difference between the two types of diagrams Fig. 1a and Fig. 1b: as the photon couples only to the stops, $\Im m d_{\tilde{\chi}^{0}}^{\gamma}$ has a smooth dependence on $\varphi_{\mu}$, whereas $\Im \mathrm{m} d_{\tilde{\chi}^{0}}^{Z}$ depends on $\varphi_{\mu}$ through the masses of the neutralinos. The thresholds of neutralino production are clearly visible at $\varphi_{\mu}=0.6 \pi$ and $\varphi_{\mu}=0.77 \pi$. Fig. $5 \mathrm{~d}$ and Fig. 5c are connected via the dispersion relations for $d^{\gamma}(s)$ and $d^{Z}(s)$.

The chargino contribution exhibits only a very small dependence on $\theta_{\tilde{b}}$ and $\varphi_{\tilde{b}}$ for the parameter region chosen. For $\varsigma_{m} d^{\gamma}(s)$ it turns out that one can neglect the neutralino contributions, so the dependence on the mixing angle $\theta_{\tilde{t}}$ and the phase $\varphi_{\tilde{t}}$ is irrelevant. However for $\Im \mathrm{m} d^{Z}(s)$ the neutralino contributions are quite big. The main contributions come from the second and the third part of eq.(35) which are proportional to $\cos 2 \theta_{\tilde{t}}$. As $\varphi_{\tilde{t}}$ does not appear in these contributions, the dependence of $d_{\tilde{\chi}^{0}}^{Z}$ on $\varphi_{\tilde{t}}$ is small.

There is a smooth dependence on $m_{\tilde{b}}$ : $\Im m d_{\tilde{\chi}^{+}}^{\gamma}$ and $\Im m d_{\tilde{\chi}^{+}}^{Z}$ increase from -0.0014 at $m_{\tilde{b}_{1}}=100 \mathrm{GeV}$ to -0.0004 at $m_{\tilde{b}_{1}}=400 \mathrm{GeV}$. The dependence of $d_{\tilde{g}}^{\gamma}$ on $m_{\tilde{t}}$ is already shown in 四. $d_{\tilde{\chi}^{0}}^{Z}$ exhibits a smooth dependence on $m_{\tilde{t}_{1}}$ : $\Im m d_{\tilde{\chi}^{0}}^{Z}$ decreases from 0.0007 at $m_{\tilde{t}_{1}}=100 \mathrm{GeV}$ to 0.0003 at $m_{\tilde{t}_{1}}=250 \mathrm{GeV}$.

In Fig. 6a we show $d^{\gamma}(s)$ and $d^{Z}(s)$ as functions of $\sqrt{s}$ where all contributions (gluino, chargino, and neutralino) are summed up. The threshold effects can be seen very clearly. There is a big enhancement in $d^{\gamma}(s)$ and $d^{Z}(s)$ because the threshold for $\tilde{\chi}_{1}^{+} \tilde{\chi}_{1}^{-}$production 
is reached at $\sqrt{s}=420 \mathrm{GeV}$ for $m_{\tilde{\chi}_{1}^{+}}=210 \mathrm{GeV}$. At $\sqrt{s}=590 \mathrm{GeV} \tilde{\chi}_{2}^{+} \tilde{\chi}_{2}^{-}$production becomes possible and again there is a big contribution but with a different sign. The additional thresholds in $d^{Z}(s)$ are due to the neutralino contributions. Fig. 6b can again be understood via dispersion relations: each spike corresponds to the opening of a new production channel.

\section{Summary and Conclusions}

We have calculated all contributions to the electric and weak dipole moment form factors of the top quark, $d^{\gamma}(s)$ and $d^{Z}(s)$, within the MSSM with complex SUSY parameters $\mu$, $A_{t}, A_{b}$, and $m_{\tilde{g}}$. These form factors can be measured by the reaction $e^{+} e^{-} \rightarrow t \bar{t}$ with $t \rightarrow b W$. They are different from zero only if $\mathrm{CP}$ is violated. After the diagonalization of the complex chargino, neutralino, stop, and sbottom mass matrices, the CP violating phases of the couplings are related to the phases of $\mu, A_{t}, A_{b}$, and $m_{\tilde{g}}$.

We find that the chargino contribution is even larger than the gluino contribution for $m_{\tilde{g}} \gtrsim 500 \mathrm{GeV}$ due to the Yukawa couplings. The neutralino exchange plays a less important role, but must not be neglected. The dependence of $d^{\gamma}(s)$ and $d^{Z}(s)$ on the SUSY parameters and on the energy is very characteristic. There are enhancements whenever the particles in the loop that couple to $\gamma, Z$ reach a threshold. Therefore by measuring the dipole moment form factors by suitable asymmetries one can get information about the SUSY parameters.

It is important to point out that we have performed our analysis within the general framework of the MSSM with complex parameters. In particular, we have not used universal SUSY parameters at the GUT scale. The numerical values of the form factors can reach about $10^{-3}$, which in general results in a measurable asymmetry of this size.

\section{Acknowledgements}

We thank Helmut Eberl for his constructive assisitance in the evaluation of the loop integrals and Sabine Kraml for the discussions about the couplings in the Lagrangian. We also thank Stefano Rigolin for his helpful correspondence regarding the numerical calculations. E.C.'s work has been supported by the Bulgarian National Science Foundation, Grant Ph510. This work was also supported by the 'Fonds zur Förderung der wissenschaftlichen Forschung' of Austria, project no. P10843-PHY. 


\section{Appendix A}

Here we show how to obtain the unitary matrices $U_{\alpha k}$ and $V_{\alpha k}$ from the complex chargino mass matrix $M_{\alpha \beta}$ eq.(18). For arbitrary $M_{\alpha \beta}$ we have

$$
M_{\alpha \beta} U_{\alpha j}^{*} V_{\beta k}^{*}=\delta_{j k} m_{k} \quad \text { and } \quad M_{\alpha \beta}=U_{\alpha k} V_{\beta k} m_{k}
$$

with unitary

$$
U_{\alpha j}^{*} U_{\alpha k}=\delta_{j k} \quad U_{\alpha k} U_{\beta k}^{*}=\delta_{\alpha \beta} \quad V_{\alpha j} V_{\alpha k}^{*}=\delta_{j k} \quad V_{\alpha k}^{*} V_{\beta k}=\delta_{\alpha \beta} \quad \text { and } m_{k} \geq 0
$$

Obtaining $V_{\alpha k}$ from

$$
\left(M_{\alpha \beta} U_{\alpha j}^{*} V_{\beta k}^{*}\right)^{*} M_{\alpha^{\prime} \beta^{\prime}} U_{\alpha^{\prime} j}^{*} V_{\beta^{\prime} l}^{*}=M_{\alpha \beta}^{*} M_{\alpha^{\prime} \gamma} \delta_{\alpha^{\prime} \alpha} V_{\beta k} V_{\gamma l}^{*}=\left(\delta_{j k} m_{k}\right)^{*} \delta_{j l} m_{l}=\delta_{k l} m_{k}^{2}
$$

one sees

$$
\delta_{k l} m_{k}=U_{\alpha k}^{*} M_{\alpha \beta} V_{\beta l}^{*}=\left(1 / m_{k}\right) \delta_{k l} m_{k}^{2}=\left(1 / m_{k}\right) M_{\alpha \gamma}^{*} V_{\gamma k} M_{\alpha \beta} V_{\beta l}^{*}
$$

that

$$
U_{\alpha k}^{*}=\left(1 / m_{k}\right) M_{\alpha \gamma}^{*} V_{\gamma k} \quad \text { or } \quad U_{\alpha k}=M_{\alpha \gamma} V_{\gamma k}^{*} / m_{k}
$$

\section{Appendix B}

Here we show how to obtain the unitary matrix $N_{\alpha k}$ from the complex symmetric neutralino mass matrix $M_{\alpha \beta}$ eq.(21) like in [14.

For arbitrary $M_{\alpha \beta}$ we have eq.(40) and (41) to obtain $u_{\alpha j}$ and $v_{\beta k}$. Because $M_{\alpha \beta}=M_{\beta \alpha}$ is symmetric $u_{\alpha k} v_{\beta k} m_{k}=u_{\beta k} v_{\alpha k} m_{k}$ or

$$
\begin{gathered}
u_{\alpha k} v_{\beta k} m_{k} \cdot v_{\alpha j}^{*} v_{\beta l}^{*}=v_{\alpha j} u_{\alpha l}^{*} m_{l} \quad u_{\beta k} v_{\alpha k} m_{k} \cdot v_{\alpha j}^{*} v_{\beta l}^{*}=u_{\beta j} v_{\beta l}^{*} m_{j} \\
\text { so } \quad v_{\alpha j}^{*} u_{\alpha l}=v_{\alpha l}^{*} u_{\alpha j}=0 \quad \text { for } \quad m_{j} \neq m_{l}
\end{gathered}
$$

so

$$
\begin{aligned}
& s_{j k}^{2}:=v_{\alpha j}^{*} u_{\alpha k}=e^{2 i \alpha_{k}} \delta_{j k} \text { with } 0 \leq \alpha_{k}<\pi \quad \text { since } \quad s_{j k}^{2 *} s_{k l}^{2}=\delta_{j l}, \\
& v_{\beta j} s_{j k}^{2}=v_{\beta j} v_{\alpha j}^{*} u_{\alpha k}=u_{\beta k} .
\end{aligned}
$$

With the definitions $s_{j k}^{1}:=e^{i \alpha_{k}} \delta_{j k}$ and $v_{\alpha k}=N_{\alpha j} s_{j k}^{1}=N_{\alpha k} e^{i \alpha_{k}}$ follows

$$
\begin{aligned}
& u_{\alpha k}=v_{\alpha j} s_{j k}^{2}=N_{\alpha j}^{*} e^{-i \alpha_{j}} e^{2 i \alpha_{k}} \delta_{j k}=N_{\alpha k}^{*} e^{i \alpha_{k}} \\
& M_{\alpha \beta}=u_{\alpha k} v_{\beta k} m_{k}=N_{\alpha k}^{*} e^{i \alpha_{k}} N_{\beta k}^{*} e^{-i \alpha_{k}} m_{k}=N_{\alpha k}^{*} N_{\beta k}^{*} m_{k} .
\end{aligned}
$$




\section{Appendix C}

Here we give the definitions of the Passarino-Veltman three point functions with the convention of [16]:

$$
\mathcal{D}^{0}=q^{2}-m_{0}^{2} \quad \text { and } \quad \mathcal{D}^{j}=\left(q+p_{j}\right)^{2}-m_{j}^{2}
$$

are the general denominators for

$$
\begin{aligned}
& C_{0}\left(p_{1}^{2},\left(p_{1}-p_{2}\right)^{2}, p_{2}^{2}, m_{0}^{2}, m_{1}^{2}, m_{2}^{2}\right):=\frac{1}{i \pi^{2}} \int d^{D} q \frac{1}{\mathcal{D}^{0} \mathcal{D}^{1} \mathcal{D}^{2}} \\
& C_{\mu}\left(p_{1}^{2},\left(p_{1}-p_{2}\right)^{2}, p_{2}^{2}, m_{0}^{2}, m_{1}^{2}, m_{2}^{2}\right):=\frac{1}{i \pi^{2}} \int d^{D} q \frac{q_{\mu}}{\mathcal{D}^{0} \mathcal{D}^{1} \mathcal{D}^{2}} \\
& =\quad p_{1 \mu} C_{1}\left(p_{1}^{2},\left(p_{1}-p_{2}\right)^{2}, p_{2}^{2}, m_{0}^{2}, m_{1}^{2}, m_{2}^{2}\right) \\
& \quad+p_{2 \mu} C_{2}\left(p_{1}^{2},\left(p_{1}-p_{2}\right)^{2}, p_{2}^{2}, m_{0}^{2}, m_{1}^{2}, m_{2}^{2}\right) \\
& C_{\mu \nu}\left(p_{1}^{2},\left(p_{1}-p_{2}\right)^{2}, p_{2}^{2}, m_{0}^{2}, m_{1}^{2}, m_{2}^{2}\right):=\frac{1}{i \pi^{2}} \int d^{D} q \frac{q_{\mu} q_{\nu}}{\mathcal{D}^{0} \mathcal{D}^{1} \mathcal{D}^{2}} \\
& =\quad g_{\mu \nu} C_{00}\left(p_{1}^{2},\left(p_{1}-p_{2}\right)^{2}, p_{2}^{2}, m_{0}^{2}, m_{1}^{2}, m_{2}^{2}\right) \\
& \quad+p_{1 \mu} p_{1 \nu} C_{11}\left(p_{1}^{2},\left(p_{1}-p_{2}\right)^{2}, p_{2}^{2}, m_{0}^{2}, m_{1}^{2}, m_{2}^{2}\right) \\
& \quad+\left(p_{1 \mu} p_{2 \nu}+p_{2 \mu} p_{1 \nu}\right) C_{12}\left(p_{1}^{2},\left(p_{1}-p_{2}\right)^{2}, p_{2}^{2}, m_{0}^{2}, m_{1}^{2}, m_{2}^{2}\right) \\
& \quad+p_{2 \mu} p_{2 \nu} C_{22}\left(p_{1}^{2},\left(p_{1}-p_{2}\right)^{2}, p_{2}^{2}, m_{0}^{2}, m_{1}^{2}, m_{2}^{2}\right) .
\end{aligned}
$$

We further use the definition

$$
C_{x}^{m, j k}=C_{x}\left(m_{t}^{2}, s, m_{t}^{2}, m_{\tilde{t}_{m}}^{2}, m_{\tilde{\chi}_{j}^{0}}^{2}, m_{\tilde{\chi}_{k}^{0}}^{2}\right) \quad x \in\{0,1,2,11,22\}
$$

and it follows

$$
C_{0}^{m, j k}=C_{0}^{m, k j} \quad C_{1}^{m, j k}=C_{2}^{m, k j} \quad C_{11}^{m, j k}=C_{22}^{m, k j}
$$

\section{Figure Captions}

Figure 1: Feynman diagrams contributing to $d^{\gamma}(s)$ and $d^{Z}(s)$ :

(a) with two fermions and one scalar in the loop

(b) with two scalars and one fermion in the loop.

Figure 2: Dependence of the masses (in $\mathrm{GeV}$ ) on $\cos \varphi_{\mu}$ for $M=230 \mathrm{GeV}$.

(a) charginos: $m_{\tilde{\chi}_{1}^{+}}$(full line), $m_{\tilde{\chi}_{2}^{+}}$(dashed line).

(b) neutralinos: $m_{\tilde{\chi}_{1}^{0}}$ (full line), $m_{\tilde{\chi}_{2}^{0}}$ (dashed line), $m_{\tilde{\chi}_{3}^{0}}$ (dotted line), $m_{\tilde{\chi}_{4}^{0}}$ (dashed-dotted line). 
Figure 3: Dependence of the chargino contribution to $d^{\gamma}(s)$ and $d^{Z}(s)$ on $|\mu|(\mathrm{GeV})$ for the reference parameter set.

(a) $\Im \mathrm{m} d_{\tilde{\chi}^{+}}^{\gamma}$ for $M=230 \mathrm{GeV}$ (full line), $M=360 \mathrm{GeV}$ (dotted line), $\Im \mathrm{m} d_{\tilde{\chi}^{+}}^{Z}$ for $M=230 \mathrm{GeV}$ (dashed line), $M=360 \mathrm{GeV}$ (dashed-dotted line).

(b) $\Re$ e $d_{\tilde{\chi}^{+}}^{\gamma}$ for $M=230 \mathrm{GeV}$ (full line), $M=360 \mathrm{GeV}$ (dotted line), $\Re$ e $d_{\tilde{\chi}^{+}}^{Z}$ for $M=230 \mathrm{GeV}$ (dashed line), $M=360 \mathrm{GeV}$ (dashed-dotted line).

Figure 4: Dependence of the neutralino contribution to $d^{\gamma}(s)$ and $d^{Z}(s)$ on $|\mu|(\mathrm{GeV})$ for the reference parameter set.

(a) $\Im \mathrm{m} d_{\tilde{\chi}^{0}}^{\gamma}$ for $M=230 \mathrm{GeV}$ (full line), $M=360 \mathrm{GeV}$ (dotted line), sm $d_{\tilde{\chi}^{0}}^{Z}$ for $M=230 \mathrm{GeV}$ (dashed line), $M=360 \mathrm{GeV}$ (dashed-dotted line).

(b) $\Re$ e $d_{\tilde{\chi}^{0}}^{\gamma}$ for $M=230 \mathrm{GeV}$ (full line), $M=360 \mathrm{GeV}$ (dotted line), $\Re$ e $d_{\tilde{\chi}^{0}}^{Z}$ for $M=230 \mathrm{GeV}$ (dashed line), $M=360 \mathrm{GeV}$ (dashed-dotted line).

Figure 5: Dependence of the chargino/neutralino contributions to $d^{\gamma}(s)$ and $d^{Z}(s)$ on $\varphi_{\mu}$ for the reference parameter set.

(a) $\Im \mathrm{m} d_{\tilde{\chi}^{+}}^{\gamma}$ for $M=230 \mathrm{GeV}$ (full line), $M=360 \mathrm{GeV}$ (dotted line), $\Im \mathrm{m} d_{\tilde{\chi}^{+}}^{Z}$ for $M=230 \mathrm{GeV}$ (dashed line), $M=360 \mathrm{GeV}$ (dashed-dotted line).

(b) $\Re$ e $d_{\tilde{\chi}^{+}}^{\gamma}$ for $M=230 \mathrm{GeV}$ (full line), $M=360 \mathrm{GeV}$ (dotted line), $\Re$ e $d_{\tilde{\chi}^{+}}^{Z}$ for $M=230 \mathrm{GeV}$ (dashed line), $M=360 \mathrm{GeV}$ (dashed-dotted line).

(c) $\Im \mathrm{m} d_{\tilde{\chi}^{0}}^{\gamma}$ for $M=230 \mathrm{GeV}$ (full line), $M=360 \mathrm{GeV}$ (dotted line), $\Im m d_{\tilde{\chi}^{0}}^{Z}$ for $M=230 \mathrm{GeV}$ (dashed line), $M=360 \mathrm{GeV}$ (dashed-dotted line).

(d) $\Re$ e $d_{\tilde{\chi}^{0}}^{\gamma}$ for $M=230 \mathrm{GeV}$ (full line), $M=360 \mathrm{GeV}$ (dotted line), $\Re$ e $d_{\tilde{\chi}^{0}}^{Z}$ for $M=230 \mathrm{GeV}$ (dashed line), $M=360 \mathrm{GeV}$ (dashed-dotted line).

Figure 6: $\quad d^{\gamma}(s)$ and $d^{Z}(s)$ for the reference parameter set with $M=230 \mathrm{GeV}$.

(a) $\Im \mathrm{m} d^{\gamma}(s)$ (full line), $\Im \mathrm{m} d^{Z}(s)$ (dashed line)

(b) $\Re$ e $d^{\gamma}(s)$ (full line), $\Re$ e $d^{Z}(s)$ (dashed line).

\section{References}

[1] CDF Collaboration, F. Abe et al, Phys.Rev.Lett. 74 (1995) 2626

D0 Collaboration, S. Abachi et al, Phys.Rev.Lett. 74 (1995) 2632

[2] W. Bernreuther and O. Nachtmann, Phys.Lett. B268 (1991) 424

A. Brandenburg, J. P. Ma, R. Münch, O. Nachtmann, Z. Phys. C51 (1991) 225

D. Atwood and A. Soni, Phys.Rev. D45 (1992) 2405, hep-ph / 9609418

W.Bernreuther, O.Nachtmann, P.Overmann, T.Schröder, Nucl.Phys. 388 (1992) 53 
C. R. Schmidt Phys.Lett. B293 (1992) 111

C. R. Schmidt and M. E. Peskin, Phys.Rev.Lett. 69 (1992) 410

W. Bernreuther and A. Brandenburg, Phys.Lett. B314 (1993) 104;

D. Chang, Wai-Yee Keung and I. Phillips, Nucl.Phys. B408 (1993) 286

B. Grzadkowski and W. Keung, Phys.Lett. B319 (1993) 526

F. Cuypers and S. Rindani, Phys.Lett. B343 (1995) 333

P. Poulose and S. Rindani, Phys.Lett. B349 (1995) 379

[3] E. Christova and M. Fabbrichesi, Phys.Lett. B315 (1993) 338

B. Grzadkowski, Phys.Lett. B305 (1993) 384

W. Bernreuter, P. Overmann, Z.Phys. C61 (1994) 599

[4] A. Bartl, E. Christova, W. Majerotto, Nucl.Phys. B460 (1996) 235; erratum Nucl.Phys. B465 (1996) 365;

[5] C.J.-C. Im, G.L. Kane and P.J. Malde, Phys.Lett. B317 (1993) 454

[6] H.E. Haber, G.L. Kane, Phys.Rep. 177 (1985) 75

J. Gunion and H. E. Haber, Nucl.Phys. B272 (1986) 1

[7] M. Dugan, B. Grinstein, L. Hall, Nucl.Phys. B255 (1985) 413

W. Bernreuter, M. Suzuki Rev.Mod.Phys. 63 (1991) 313

W. Hollik, J.I. Illana, S. Rigolin, D. Stöckinger, hep-ph / 9711322

[8] Y. Kizukuri and N. Oshimo, Phys.Rev. D45 (1992) 1806

[9] J.M. Ortega, Matrix Theory (Plenum Press, New York 1987)

[10] R. Garisto, J.D. Wells, Phys.Rev. D55 (1997) 1611

[11] C. Hamzaoui, M. Pospelov, R. Roiban, Phys.Rev. D56 (1997) 4295

[12] Y. Kizukuri and N. Oshimo, Phys.Rev. D46 (1992) 3025

[13] S. Kraml, H. Eberl, A. Bartl, W. Majerotto, W. Porod, Phys.Lett. B386 (1996) 175

[14] S. M. Bilenky, S. T. Petcov, Rev.Mod.Phys. 59, No. 3, Part 1 (1987) 671

[15] G. Passarino and M. Veltman, Nucl.Phys. B160 (1979) 151

[16] A. Denner, Progress of Physics 41 (1993) 4, 307 


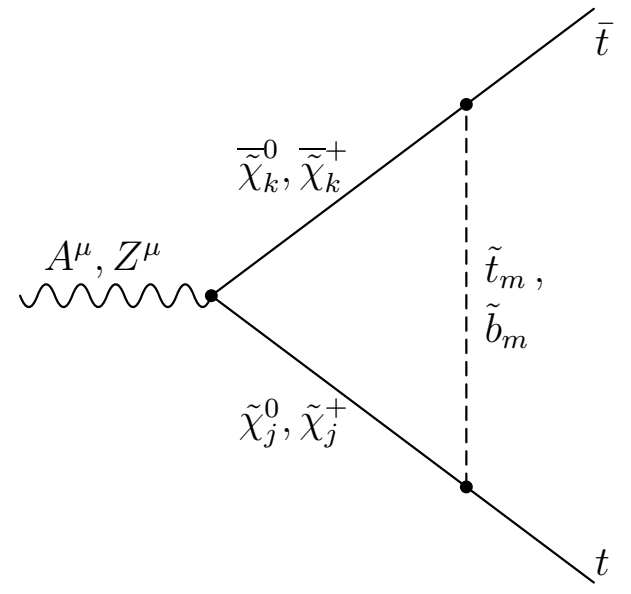

Fig. 1a

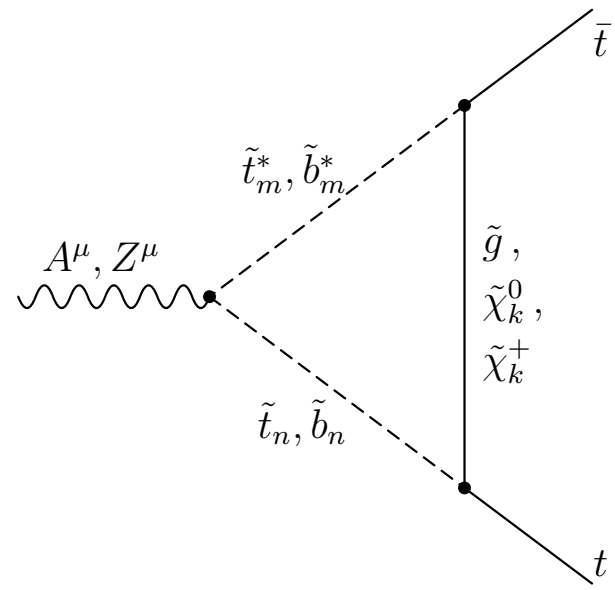

Fig. 1b

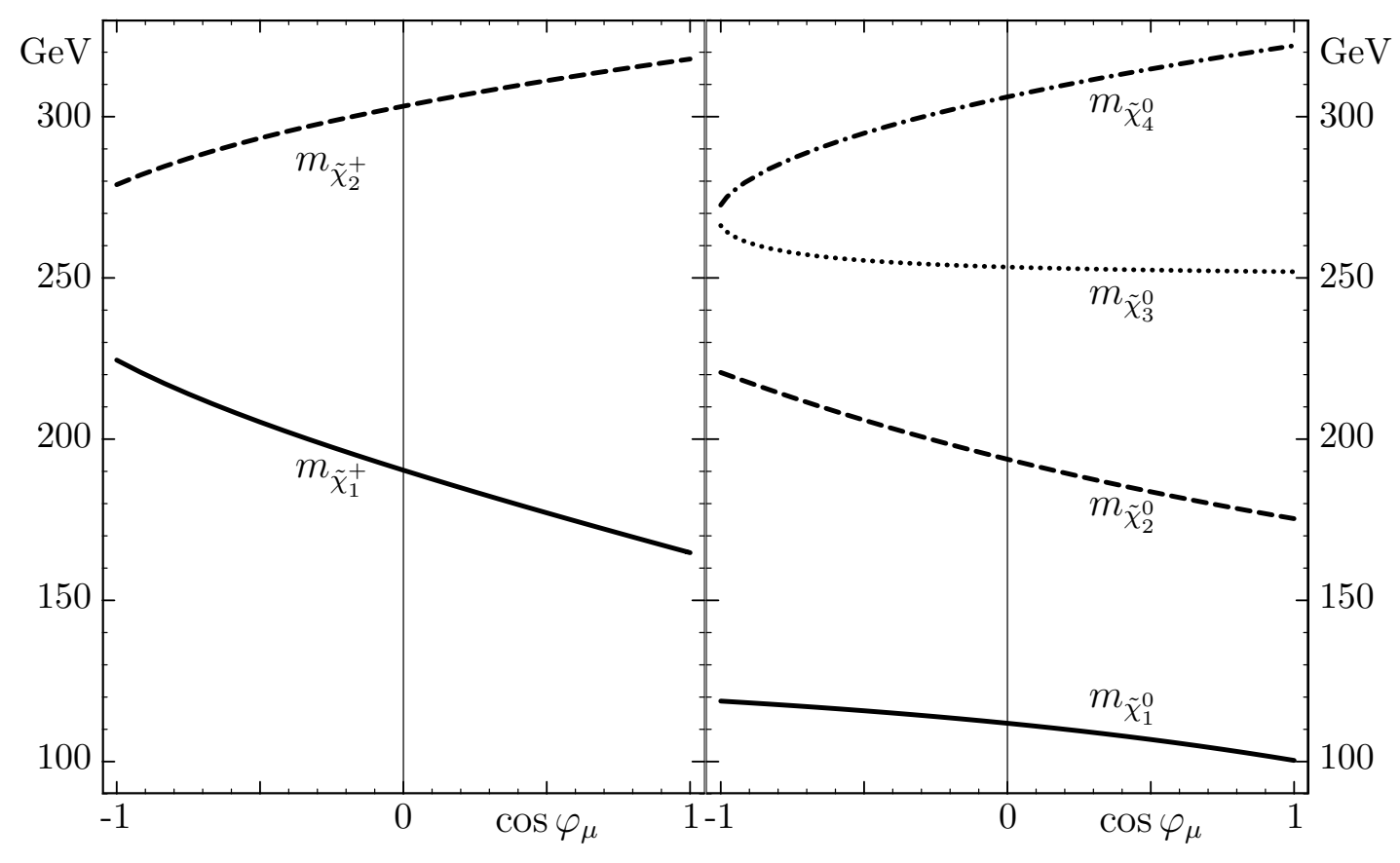

Fig. 2a

Fig. 2b 


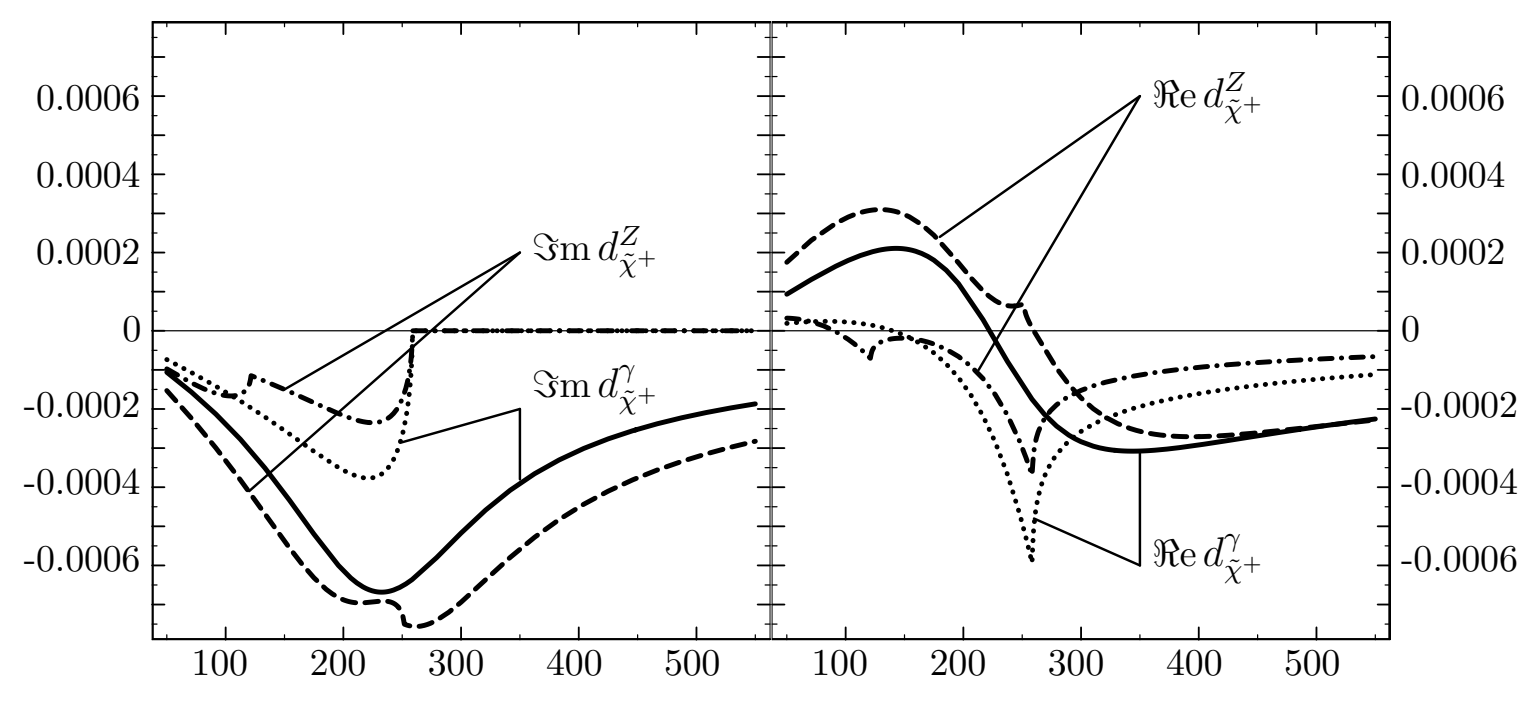

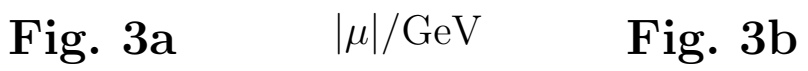

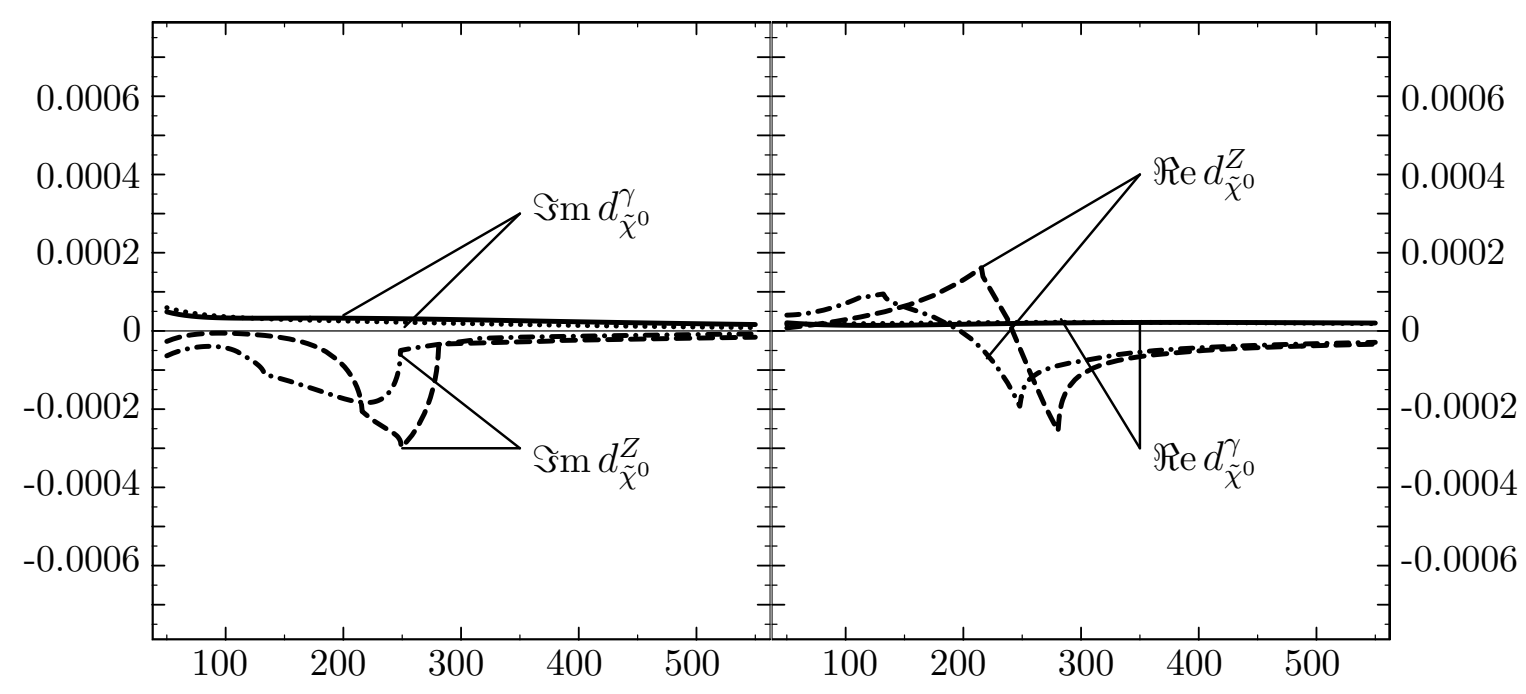

Fig. 4a $\quad|\mu| / \mathrm{GeV} \quad$ Fig. 4b 


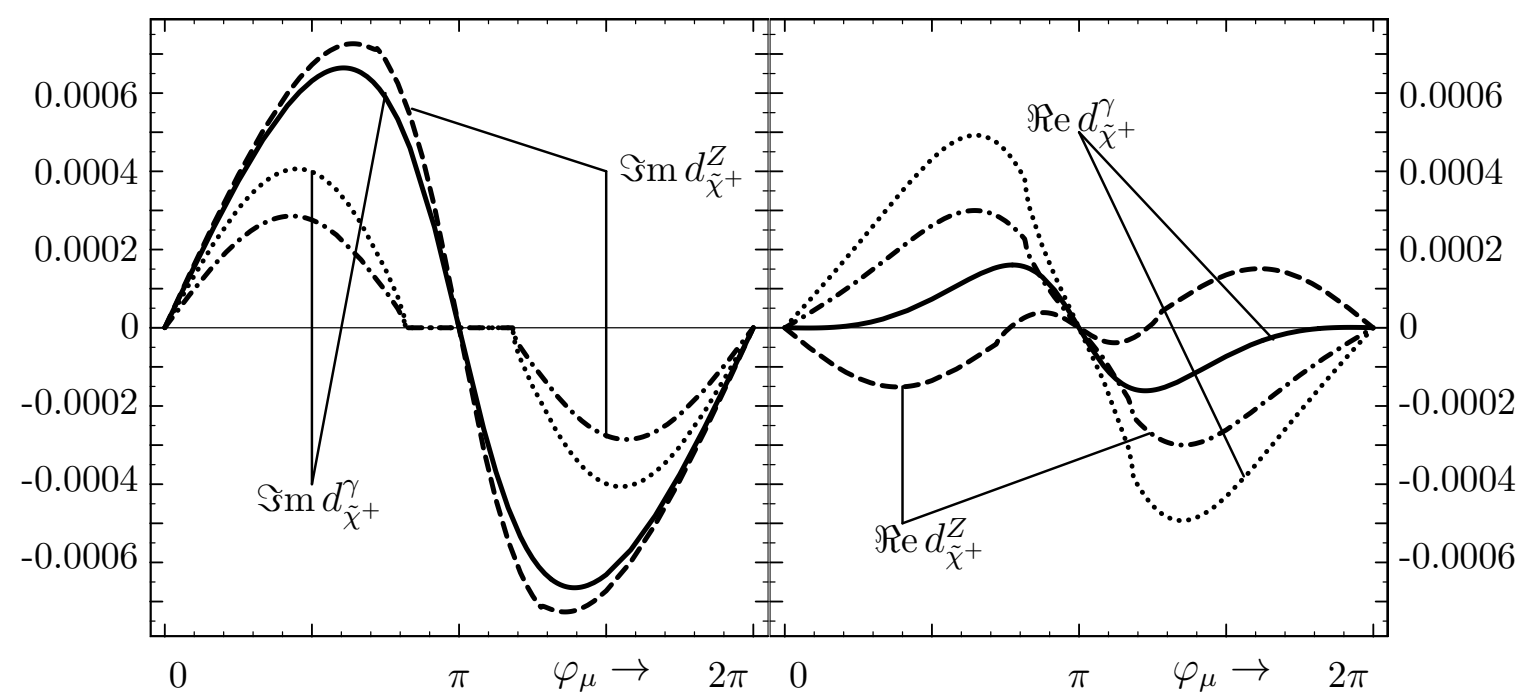

Fig. 5a

Fig. 5b

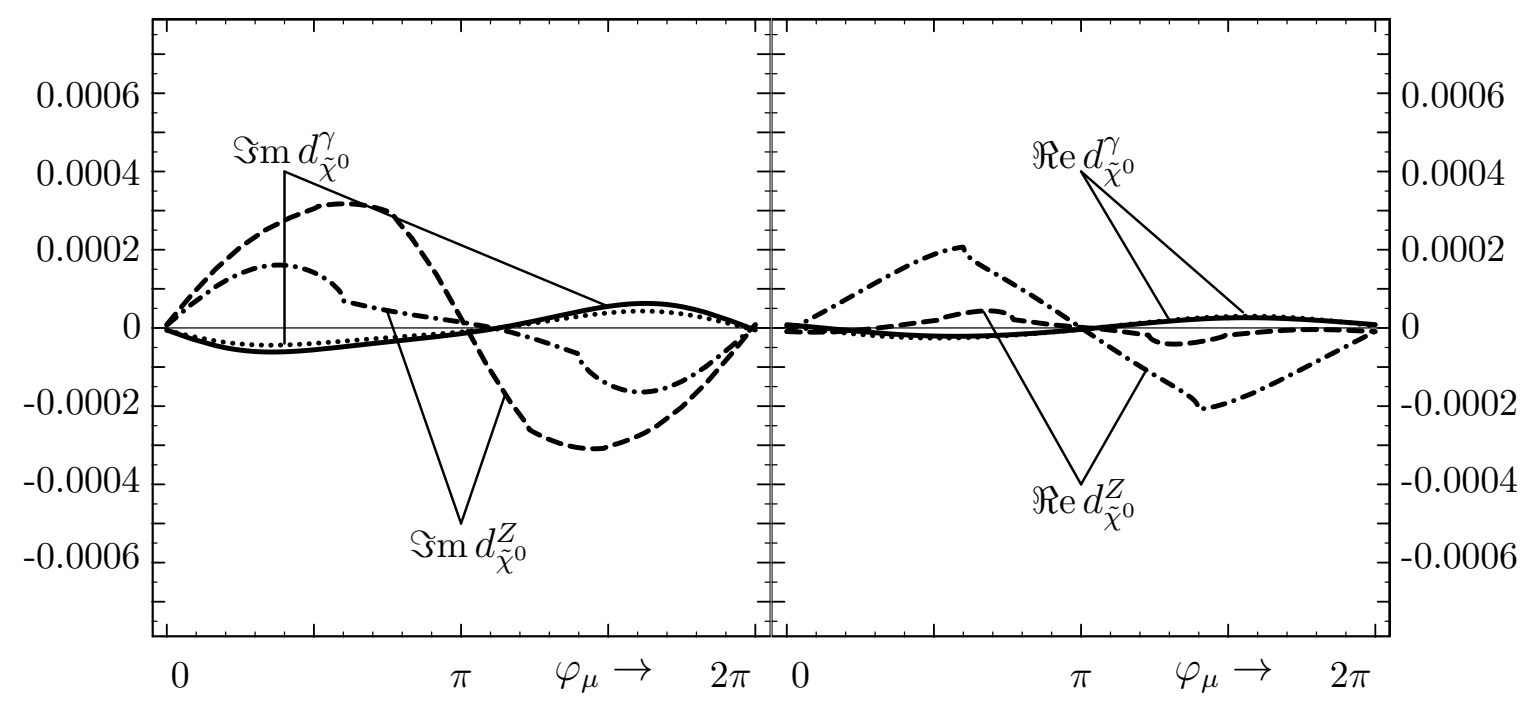

Fig. 5c

Fig. 5d 


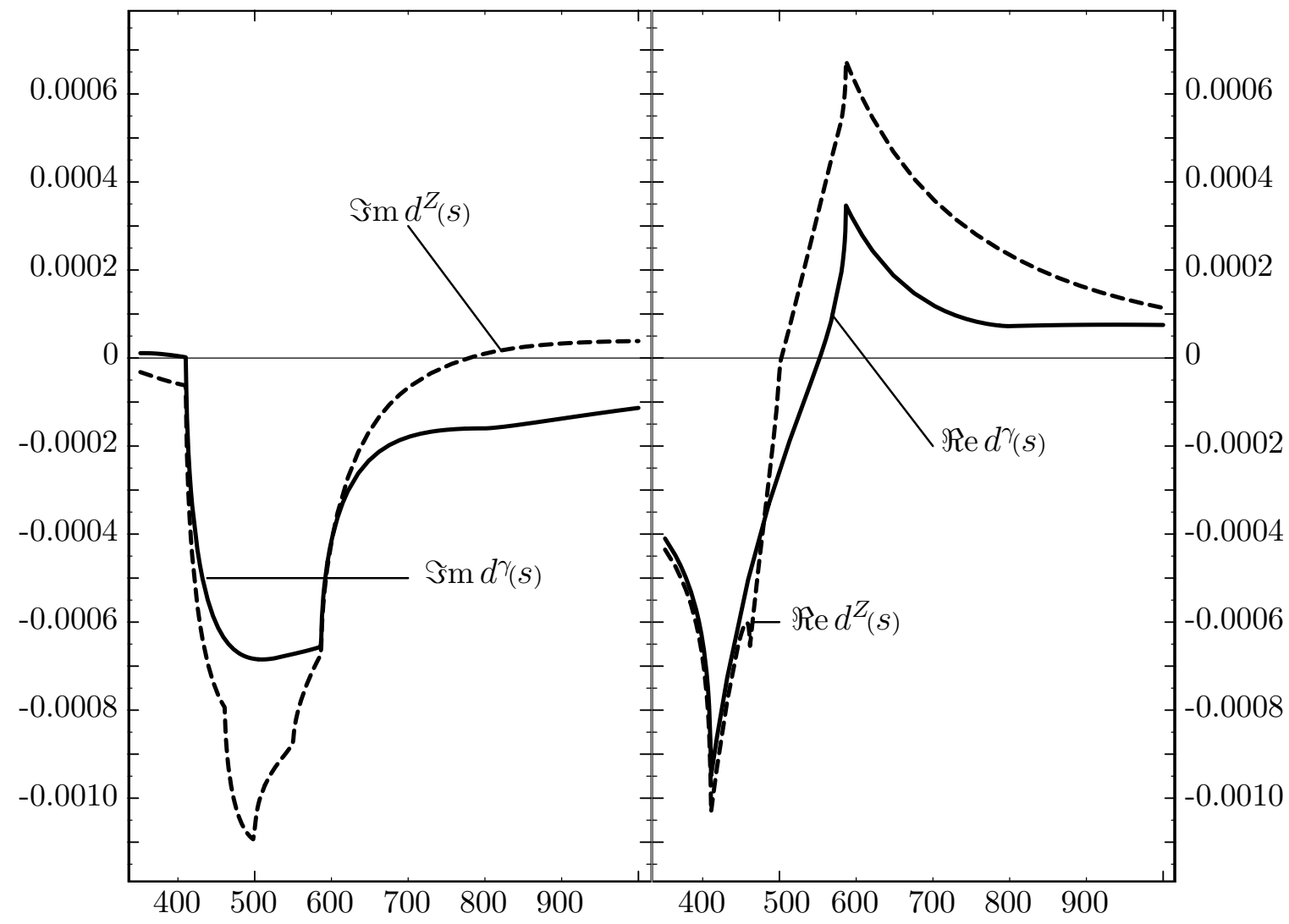

Fig. 6a $\quad \sqrt{s} / \mathrm{GeV} \quad$ Fig. 6b 


\section{Figure Captions for $\tan \beta=3$}

Figure $\overline{2}: \quad$ Dependence of the masses (in $\mathrm{GeV}$ ) on $\cos \varphi_{\mu}$ for $M=230 \mathrm{GeV}$.

(a) charginos: $m_{\tilde{\chi}_{1}^{+}}$(full line), $m_{\tilde{\chi}_{2}^{+}}$(dashed line).

(b) neutralinos: $m_{\tilde{\chi}_{1}^{0}}$ (full line), $m_{\tilde{\chi}_{2}^{0}}$ (dashed line), $m_{\tilde{\chi}_{3}^{0}}$ (dotted line), $m_{\tilde{\chi}_{4}^{0}}$ (dashed-dotted line).

Figure $\overline{3}$ : Dependence of the chargino contribution to $d^{\gamma}(s)$ and $d^{Z}(s)$ on $|\mu|(\mathrm{GeV})$ for the reference parameter set.

(a) $\Im \mathrm{m} d_{\tilde{\chi}^{+}}^{\gamma}$ for $M=230 \mathrm{GeV}$ (full line), $M=360 \mathrm{GeV}$ (dotted line), $\Im \mathrm{m} d_{\tilde{\chi}^{+}}^{Z}$ for $M=230 \mathrm{GeV}$ (dashed line), $M=360 \mathrm{GeV}$ (dashed-dotted line).

(b) $\Re$ e $d_{\tilde{\chi}^{+}}^{\gamma}$ for $M=230 \mathrm{GeV}$ (full line), $M=360 \mathrm{GeV}$ (dotted line), $\Re$ e $d_{\tilde{\chi}^{+}}^{Z}$ for $M=230 \mathrm{GeV}$ (dashed line), $M=360 \mathrm{GeV}$ (dashed-dotted line).

Figure $\overline{4}$ : Dependence of the neutralino contribution to $d^{\gamma}(s)$ and $d^{Z}(s)$ on $|\mu|(\mathrm{GeV})$ for the reference parameter set.

(a) $\Im m d_{\tilde{\chi}^{0}}^{\gamma}$ for $M=230 \mathrm{GeV}$ (full line), $M=360 \mathrm{GeV}$ (dotted line), $\Im m d_{\tilde{\chi}^{0}}^{Z}$ for $M=230 \mathrm{GeV}$ (dashed line), $M=360 \mathrm{GeV}$ (dashed-dotted line).

(b) $\Re$ e $d_{\tilde{\chi}^{0}}^{\gamma}$ for $M=230 \mathrm{GeV}$ (full line), $M=360 \mathrm{GeV}$ (dotted line), $\Re$ e $d_{\tilde{\chi}^{0}}^{Z}$ for $M=230 \mathrm{GeV}$ (dashed line), $M=360 \mathrm{GeV}$ (dashed-dotted line).

Figure $\overline{5}$ : Dependence of the chargino/neutralino contributions to $d^{\gamma}(s)$ and $d^{Z}(s)$ on $\varphi_{\mu}$ for the reference parameter set.

(a) $\Im \mathrm{m} d_{\tilde{\chi}^{+}}^{\gamma}$ for $M=230 \mathrm{GeV}$ (full line), $M=360 \mathrm{GeV}$ (dotted line), $\Im \mathrm{m} d_{\tilde{\chi}^{+}}^{Z}$ for $M=230 \mathrm{GeV}$ (dashed line), $M=360 \mathrm{GeV}$ (dashed-dotted line).

(b) $\Re$ e $d_{\tilde{\chi}^{+}}^{\gamma}$ for $M=230 \mathrm{GeV}$ (full line), $M=360 \mathrm{GeV}$ (dotted line), $\Re$ e $d_{\tilde{\chi}^{+}}^{Z}$ for $M=230 \mathrm{GeV}$ (dashed line), $M=360 \mathrm{GeV}$ (dashed-dotted line).

(c) $\Im \mathrm{m} d_{\tilde{\chi}^{0}}^{\gamma}$ for $M=230 \mathrm{GeV}$ (full line), $M=360 \mathrm{GeV}$ (dotted line), $\Im \mathrm{m} d_{\tilde{\chi}^{0}}^{Z}$ for $M=230 \mathrm{GeV}$ (dashed line), $M=360 \mathrm{GeV}$ (dashed-dotted line).

(d) $\Re$ e $d_{\tilde{\chi}^{0}}^{\gamma}$ for $M=230 \mathrm{GeV}$ (full line), $M=360 \mathrm{GeV}$ (dotted line), $\Re$ e $d_{\tilde{\chi}^{0}}^{Z}$ for $M=230 \mathrm{GeV}$ (dashed line), $M=360 \mathrm{GeV}$ (dashed-dotted line).

Figure $\overline{6}: \quad d^{\gamma}(s)$ and $d^{Z}(s)$ for the reference parameter set with $M=230 \mathrm{GeV}$.

(a) $\Im m d^{\gamma}(s)$ (full line), $\Im m d^{Z}(s)$ (dashed line)

(b) $\Re$ e $d^{\gamma}(s)$ (full line), $\Re$ e $d^{Z}(s)$ (dashed line). 


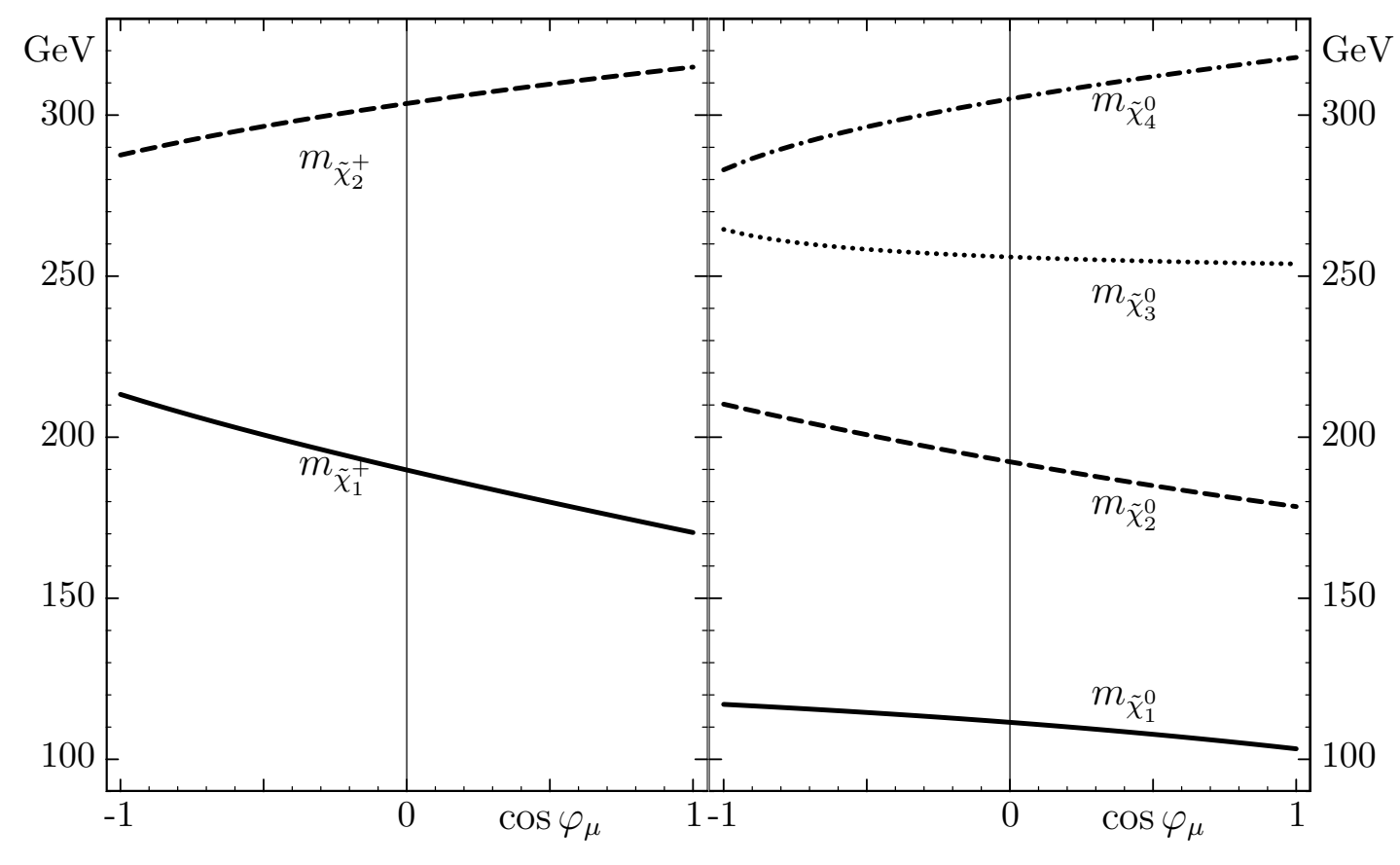

Fig. $\overline{2} \mathbf{a}$

Fig. $\overline{2}$ b 


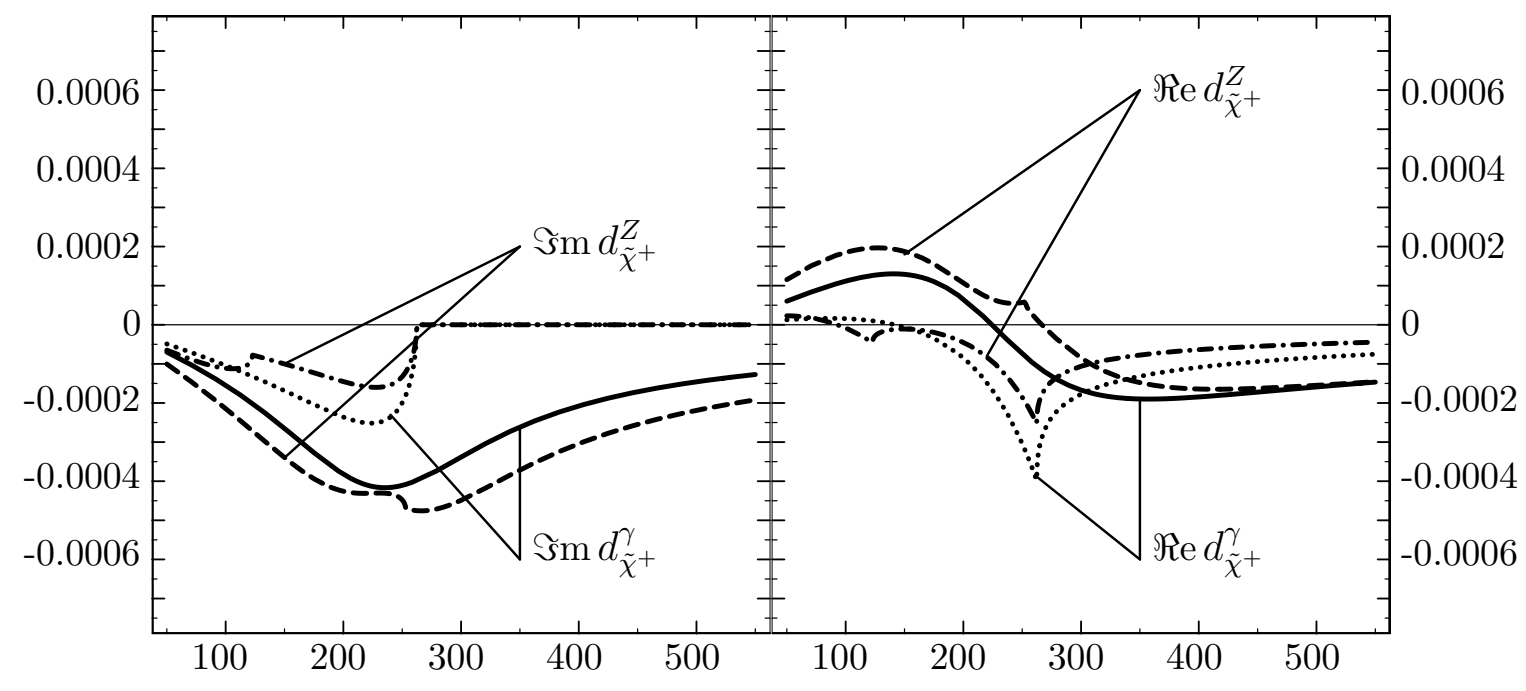

$\begin{array}{lll}\text { Fig. } \overline{3} \mathbf{a} & |\mu| / \mathrm{GeV} & \text { Fig. } \overline{3} \mathbf{b}\end{array}$

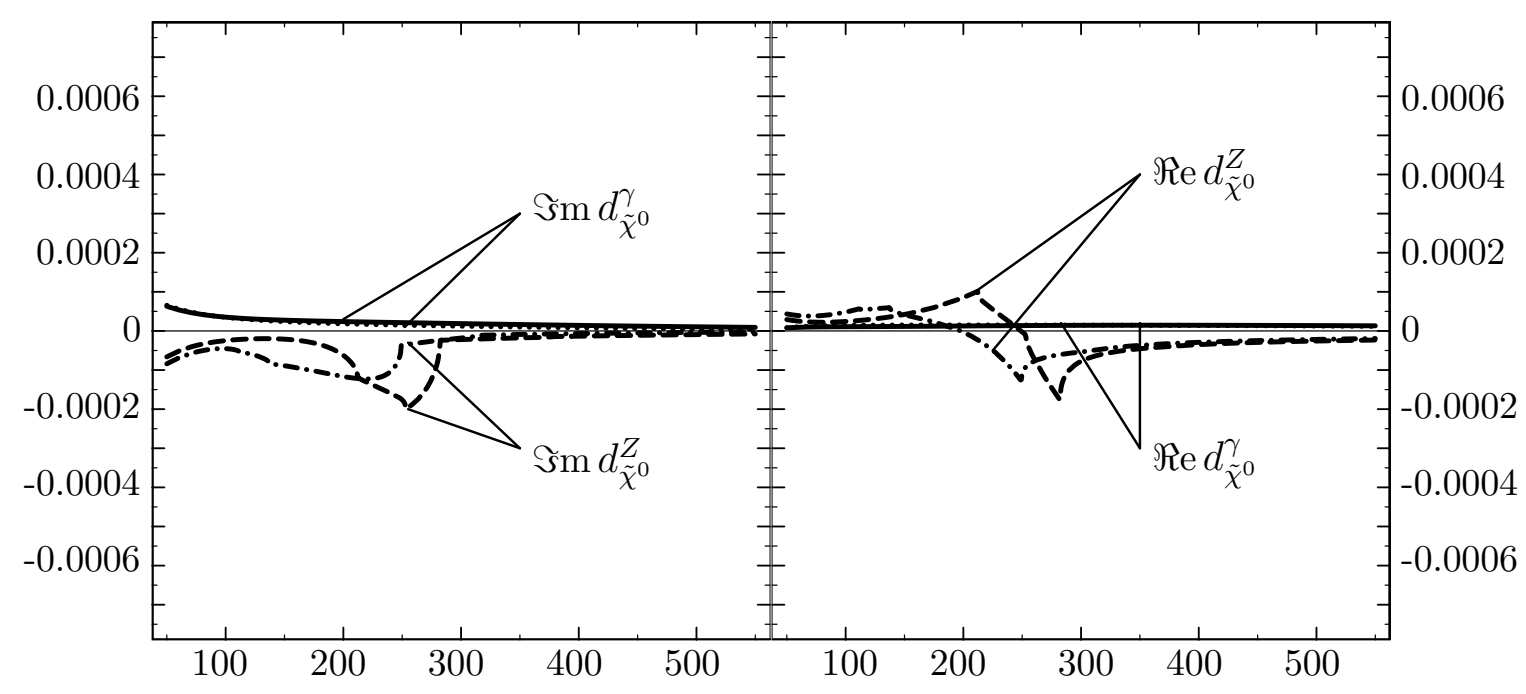

$\begin{array}{lll}\text { Fig. } \overline{4} \mathbf{a} & |\mu| / \mathrm{GeV} & \text { Fig. } \overline{4} \mathbf{b}\end{array}$ 


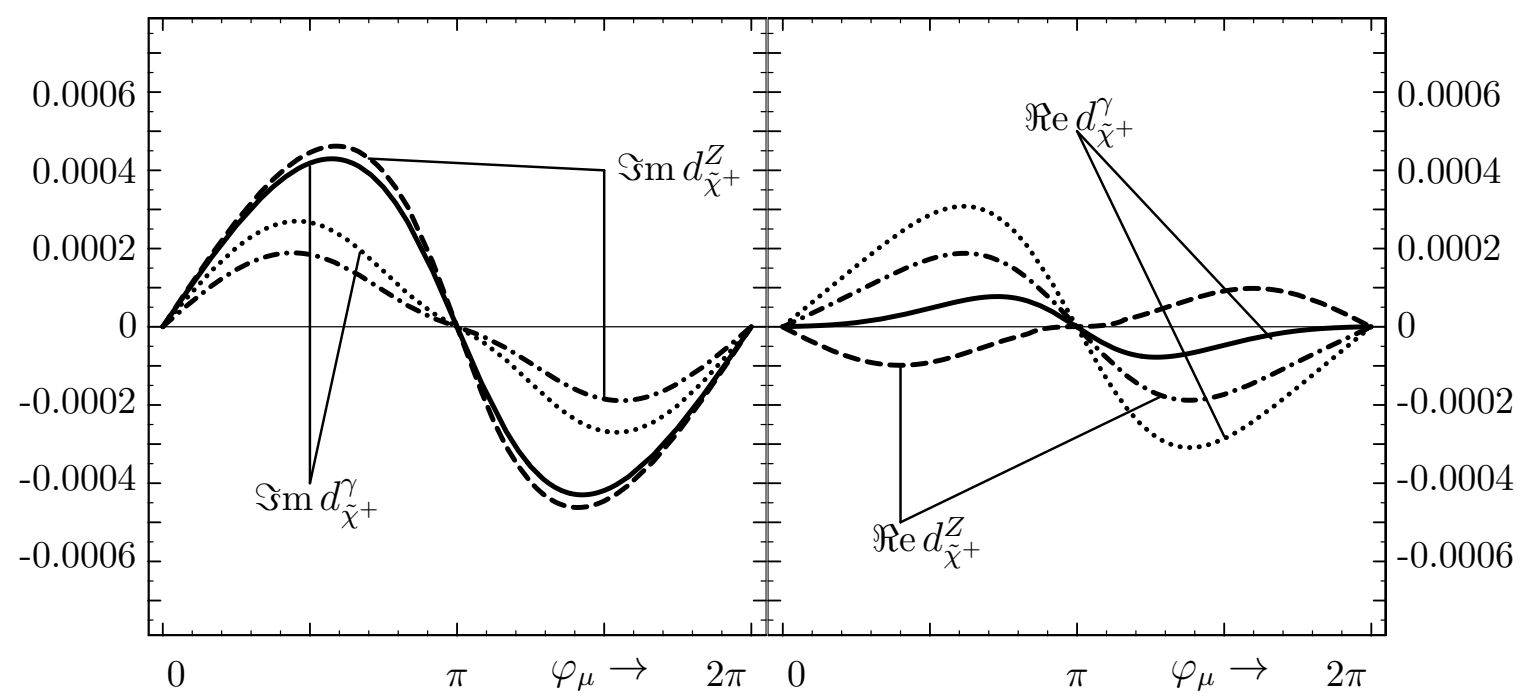

Fig. $\overline{5} \mathbf{a}$

Fig. $\overline{5} \mathbf{b}$

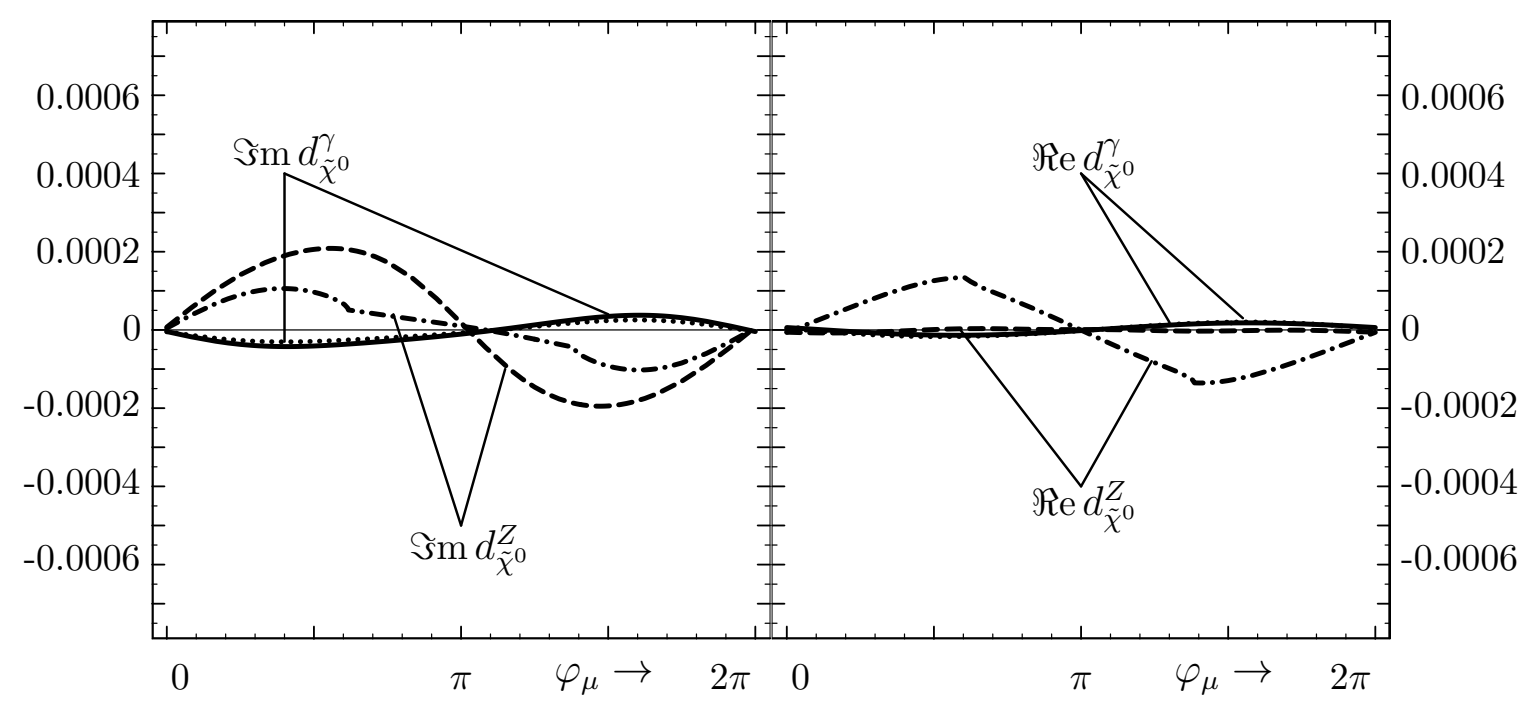

Fig. $\overline{5} \mathbf{c}$

Fig. $\overline{5} \mathrm{~d}$ 


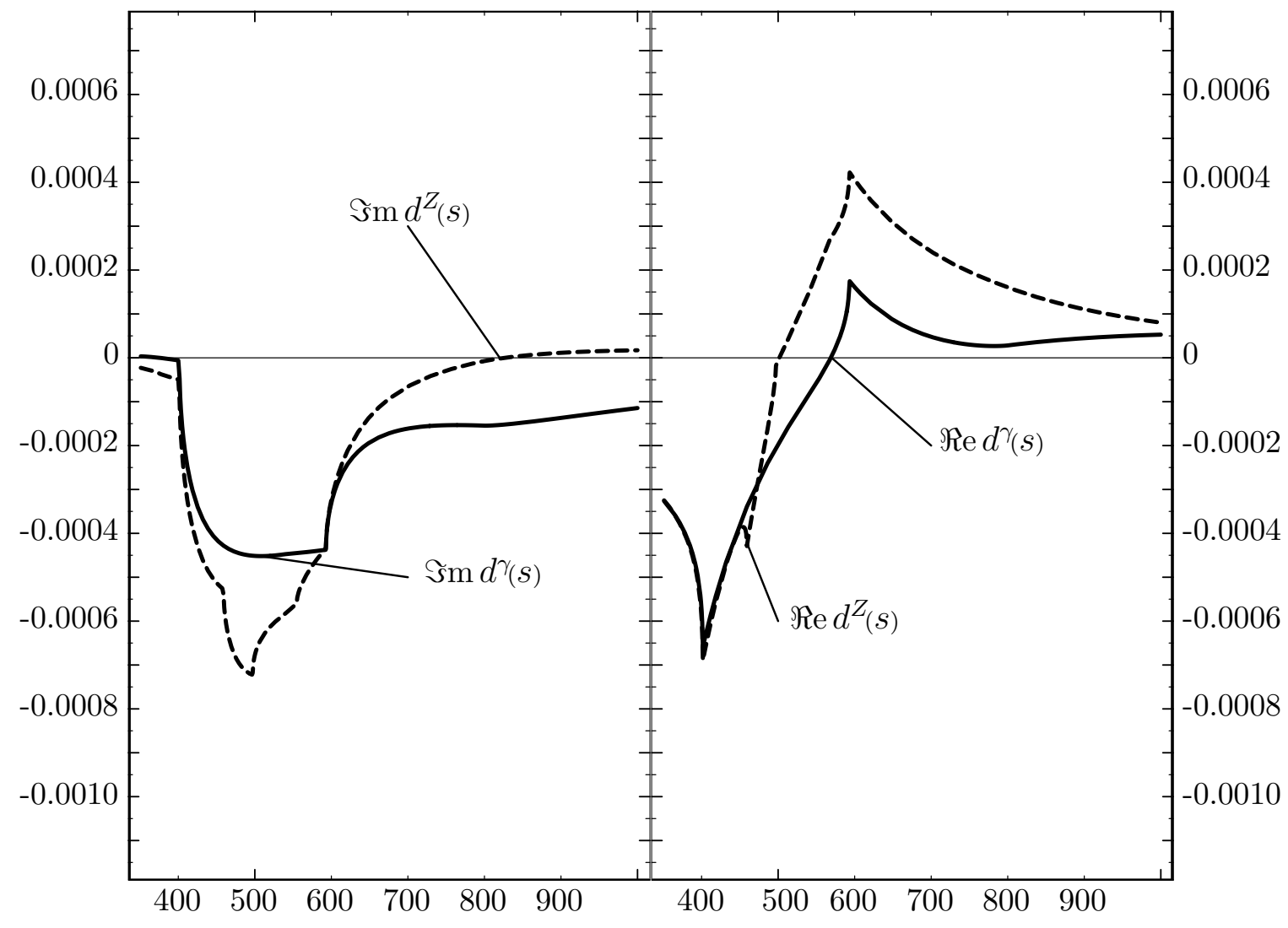

$\begin{array}{lll}\text { Fig. } \overline{6} \mathbf{a} & \sqrt{s} / \mathrm{GeV} & \text { Fig. } \overline{6} \mathbf{b}\end{array}$ 